Check for updates

Cite this: RSC Adv., 2018, 8, 25617

Received 20th April 2018

Accepted 30th June 2018

DOI: $10.1039 / \mathrm{c} 8 \mathrm{ra03408g}$

rsc.li/rsc-advances

\section{A study on the removal of propyl, butyl, and benzyl parabens via newly synthesised ionic liquid loaded magnetically confined polymeric mesoporous adsorbent $\dagger$}

\author{
Masrudin Md Yusoff, ${ }^{a}$ Noorfatimah Yahaya, ${ }^{a}$ Noorashikin Md Saleh ${ }^{b}$ \\ and Muggundha Raoov (D) *cd
}

This study investigated the effectiveness of ionic liquids (ILs) loaded onto the surface of a polymeric adsorbent ( $\beta C D$-TDI) grafted with modified magnetic nanoparticles (MNPs) via an analysis of water treatment, which resulted in high removal of selected endocrine-disrupting chemicals (parabens). The syntheses of MNPS, MNP- $\beta C D-T D I$, and IL-MNP- $\beta C D$-TDI were characterised and compared using Fourier transform infrared (FT-IR) spectroscopy, carbon-hydrogen-nitrogen (CHN) analysis, vibrating sample magnetometry (VSM), scanning electron microscopy (SEM), transmission electron microscopy (TEM), the Brunauer-EmmettTeller (BET) method, thermogravimetric analysis (TGA), and X-ray diffraction (XRD). The results of SEM and TEM indicated that the pore size distribution exhibited mesoporous characteristics with a small surface area (BET analysis: $42.95 \mathrm{~m}^{2} \mathrm{~g}^{-1}$ ). Furthermore, a preliminary sorption experiment demonstrated the ability of ILMNP- $\beta C D-T D I$ to enhance not only the sorption capacity, but also the removal of propyl paraben (PP), butyl paraben (BP), and benzyl paraben (ArP). The adsorption process appeared to be $\mathrm{pH}$-dependent, and hence the optimum $\mathrm{pH}$ of 6 was selected for a subsequent batch adsorption study of all the studied parabens with an equilibrium time of $80 \mathrm{~min}$. Next, in an attempt to investigate the interactions that occur between the adsorbent and the adsorbates, adsorption kinetics and isotherm studies were performed. All the studied parabens were found to best fit pseudo-second-order kinetics and the Freundlich isotherm with $R^{2}>0.98$ at room temperature $(298 \mathrm{~K})$. The interaction of the host-guest inclusion complex and the $\pi-\pi$ interaction between $\beta C D$ and a selected paraben compound (ArP) were identified by performing ${ }^{1} \mathrm{H}$ nuclear magnetic resonance (NMR), together with ultraviolet-visible (UV-vis) spectroscopic analysis. Finally, the adsorption efficiency of the developed material was practically tested on tap water, drain water, and industrial wastewater, which revealed a significant removal of parabens of up to $60-90 \%$ in comparison with a prior analysis.

\section{Introduction}

Magnetic nanoparticles (MNPs) have appeared to have great potential as sorbents in a number of applications studied by researchers. Besides, they have the ability to amalgamate easily

${ }^{a}$ Integrative Medicine Cluster, Advanced Medical and Dental Institute, Universiti Sains Malaysia, Bertam, 13200 Kepala Batas, Penang, Malaysia

${ }^{b}$ Research Centre For Sustainable Process Technology, Chemical Engineering Programme, Faculty of Engineering and Built Environment, Universiti Kebangsaan Malaysia, 43600 UKM Bangi, Selangor, Malaysia

'University of Malaya Centre for Ionic Liquids, Department of Chemistry, Faculty of Science, Universiti Malaya, Kuala Lumpur 50603, Malaysia. E-mail: muggundha@ um.edu.my

${ }^{d}$ Department of Chemistry, Faculty of Science, Universiti Malaya, Kuala Lumpur 50603, Malaysia

$\dagger$ Electronic supplementary information (ESI) available. See DOI: $10.1039 / \mathrm{c} 8 \mathrm{ra03408g}$ with any pollutant in huge amounts. ${ }^{1}$ In fact, the term MNPs refers to a class of nanoparticle agglomerates, which resemble particles that are small in size and can be manipulated using an external magnetic field. ${ }^{2}$ In addition, MNPs have been widely employed by researchers owing to their nanoscale sizes, which are typically around $10-20 \mathrm{~nm}$ and correspond to those of mesoporous types of materials. Nanomaterials or nanoparticles are materials with two or more dimensions and unique size dependence in terms of physical and chemical properties. ${ }^{3}$ Therefore, in order to develop a new promising adsorbent, MNPs alone are insufficient to maximize the adsorption capacity for targeted analytes and require several modifications of the surface of MNPs with other materials. ${ }^{4-12}$ This may enable exceptional outcomes, especially when dealing with small sample volumes, owing to the large surface areas. ${ }^{13}$

Furthermore, the growing interest in the field of supramolecular chemistry has allowed this study to be carried out. As 
such, this study represents an experimental study in which MNPs were coated with cyclodextrin (CD) as an effective adsorbent for the removal of parabens. CDs, which are also known as cycloamyloses, are a family of compounds that are composed of sugars bound together in a ring (cyclic oligosaccharides). The widely used applications of CDs in pharmaceuticals, food, drug delivery, and chemicals are highly desirable owing to the unique characteristics of their structure. CDs are produced by cyclomaltodextrin glucanotransferase, whereby three common cyclodextrins are available with 6,7 or 8 D-glucopyranosyl residues, namely, $\alpha$-CD ( 6 glucose units), $\beta$-CD (7 glucose units), and $\gamma$-CD (8 glucose units), respectively, which are linked in a ring by $\alpha$-1,4-glycosidic linkages. ${ }^{14}$ As such, $\beta$ cyclodextrin $(\beta C D)$ is a natural starch derived from molecules that is a torus-shaped cyclic oligosaccharide with an internal hydrophobic cavity. ${ }^{15}$

$\beta C D$ was selected for the experimental work in this study because it is low in cost and has the ability to form solid inclusion complexes ${ }^{\mathbf{1 6 , 1 7}}$ via various kinds of interactions ${ }^{18}$ with different types of guest compounds. Furthermore, $\beta C D$ can alter its properties via polymerization upon cross-linking, which refers to the process of chemically joining two or more molecules by a covalent bond and transforming the molecule into a water-insoluble molecule. ${ }^{19} \beta \mathrm{CD}$ can also be cross-linked by a reaction between hydroxyl groups on the chain and a coupling agent to form a water-insoluble network. ${ }^{20,21}$ Owing to the properties of $\mathrm{CD}$, which contains many hydroxyl groups, the best cross-linking agent is a diisocyanate linker such as toluene 2,4-diisocyanate (TDI) ${ }^{22-26}$ because of its reactivity towards hydroxyl groups. Hence, TDI was selected to be used in this study in order to transform the $\beta C D$ molecule into a threedimensional network polymer ( $\beta$ CD-TDI).

Furthermore, CD polymers have also emerged as an area of interest among academicians, especially in combination with $\mathrm{MNPs}^{27-35}$ such as those with the formula $\mathrm{Fe}_{3} \mathrm{O}_{4} \cdot{ }^{36}$ MNP surface modification appears to be a frequently used technique for retaining their internal superior magnetic properties upon combination with polymeric adsorbents. Therefore, the aggregation of MNPs and the transformation of magnetite $\left(\mathrm{Fe}_{3} \mathrm{O}_{4}\right)$ into maghemite $\left(\gamma-\mathrm{Fe}_{2} \mathrm{O}_{3}\right)$ are attributable to the reaction of $\mathrm{Fe}(\mathrm{II})$ cations with oxygen, which can be prevented. ${ }^{29}$ The unique characteristics of combinations of these types of adsorbents are the inner MNP itself, which possesses the ability to sense and respond to an external magnetic field, whereas the outer CD polymer functions as an inclusion site and a specific container for the adsorption of targeted analytes. Thus, the modification of MNPs with a $\beta C D$ polymer seems to provide an effective adsorbent for the removal of organic compounds.

In addition, in order to increase the selectivity for targeted analytes, the introduction of an ionic liquid (IL) onto a polymer surface has sparked interest in a number of studies. In conjunction with the properties of $\beta C D$, a number of methods have been developed that promote the incorporation of $\beta C D$ with an IL, mainly because the presence of an IL has been proven to enable more chemical interactions between targeted analytes. ${ }^{37-42}$ The term IL refers to a type of salt in the form of a liquid below $100{ }^{\circ} \mathrm{C}$ or even at room or ambient temperature, of which the latter are also known as room-temperature ionic liquids (RTILs). ${ }^{43}$ RTILs have begun to gain wide recognition as novel solvents within the field of chemistry owing to the unique non-volatility, non-flammability, low viscosity, and electrochemical stability of ILs, which are beneficial in various types of applications, especially in supramolecular materials. ${ }^{\mathbf{4 4 , 4 5}}$ Therefore, a new approach has been developed in this study by loading an IL onto the surface of MNP- $\beta C D$-TDI so as to create a new magnetically confined polymeric mesoporous adsorbent loaded with an ionic liquid (IL-MNP- $\beta$ CD-TDI).

The newly developed material can be used to remove endocrine-disrupting chemicals (propyl, butyl, and benzyl parabens) from the environment, especially emerging pollutants ${ }^{\mathbf{4 6}}$ that may cause serious diseases such as cancer. ${ }^{47}$ Contamination of environmental bodies of water has always been associated with uncontrolled disposal of wastewater from industries, which has an adverse impact upon the health of organisms. ${ }^{48-50}$ Therefore, adsorption techniques that integrate such a novel approach can be evaluated via an adsorption process. This study has been divided into three principal parts, namely, (1) synthesis of adsorbents, (2) characterisation of adsorbents, and (3) batch removal experiments. The adsorption process was optimised in order to ensure exceptional adsorption of the targeted analytes, as well as to study the mechanism of the interaction between the adsorbent and the adsorbates. Finally, the applicability of the adsorbent was confirmed by treating real environmental water samples.

\section{Experimental}

\subsection{Materials and chemicals}

Standard samples of three paraben compounds, namely, propyl paraben (PP), butyl paraben (BP), and benzyl paraben (ArP), 1butyl-3-methylimidazolium chloride (BMIM-Cl), toluene-2,4diisocyanate (TDI), and anhydrous dimethyl sulfoxide- $\mathrm{d}_{6}$ (DMSO) were purchased from Sigma-Aldrich (St. Louis, MO, USA). Iron(II) chloride tetrahydrate $\left(\mathrm{FeCl}_{2} \cdot 4 \mathrm{H}_{2} \mathrm{O}\right)$ and iron(III) chloride hexahydrate $\left(\mathrm{FeCl}_{3} \cdot 6 \mathrm{H}_{2} \mathrm{O}\right)$ were purchased from $\mathrm{R} \& \mathrm{M}$ Chemicals (Essex, UK), whereas $\beta$-cyclodextrin ( $\beta C D, 99 \%)$ was commercially available and was purchased from Acros (Hungary). Moreover, acetonitrile (ACN), methanol (MeOH) (HPLC grade, 99.7\%), acetone (technical grade), and ammonia solution (25\%) were supplied by Friendemann Schmidt (Parkwood, Australia), whereas anhydrous $N, N$-dimethylformamide (DMF) was purchased from Merck (Kenilworth, NJ, USA). Analyticalgrade absolute ethanol (denatured, 99.7\%) was purchased from J. Kollin Chemicals (Midlothian, UK), whereas deionised (DI) water (18.2 M $\Omega \mathrm{cm}$ ) was provided by a Sartorius Milli-Q system (Aubagne, France).

\subsection{Part 1: Synthesis of adsorbents}

The adsorbents were prepared in accordance with a procedure detailed in a prior work. ${ }^{51}$ Co-precipitation was performed under a non-oxidising nitrogen atmosphere in order to generate bare MNPs with a molar ratio of $1: 2$. Next, $\mathrm{FeCl}_{2} \cdot 4 \mathrm{H}_{2} \mathrm{O}(0.86 \mathrm{~g})$ and $\mathrm{FeCl}_{3} \cdot 6 \mathrm{H}_{2} \mathrm{O}(2.34 \mathrm{~g})$ were dissolved in $40 \mathrm{~mL}$ of deionised 
water and stirred for $30 \mathrm{~min}$ at $1200 \mathrm{rpm}$. The solution temperature was increased to $90{ }^{\circ} \mathrm{C}$ and $5 \mathrm{~mL}$ of $\mathrm{NH}_{4} \mathrm{OH}(25 \%)$ was added directly, which was then stirred for an hour. In order to remove any unreacted chemicals, the nanoparticles that formed were washed with deionised water five or six times. The resulting product was isolated using an external magnet and dried in a vacuum oven at $40{ }^{\circ} \mathrm{C}$.

Next, a CD polymer was synthesised in accordance with a literature method. ${ }^{37,39} \beta \mathrm{CD}(2 \mathrm{~g}, 1.76 \mathrm{mmol})$ was dissolved in $50 \mathrm{~mL}$ of anhydrous DMF at room temperature. In an inert atmosphere, the TDI linker $(2.54 \mathrm{~mL}, 17.6 \mathrm{mmol})$ was added and the reaction mixture was stirred for $24 \mathrm{~h}$ at $70{ }^{\circ} \mathrm{C}$. The addition of excess acetone caused the precipitation of $\beta C D$-TDI. In order to remove residual DMF from the polymer, the precipitate that formed was stirred and allowed to settle in acetone for $10 \mathrm{~min}$. Then, the reaction mixture was filtered and washed with acetone and deionised water several times to remove unreacted cross-linker, and was later dried overnight in a vacuum.

After the drying process, MNP- $\beta$ CD-TDI was synthesised via a one-step co-precipitation method ${ }^{\mathbf{3 4 5 2}}$ by dissolving $\mathrm{FeCl}_{2}$ $\cdot 4 \mathrm{H}_{2} \mathrm{O}$ (0.86 g, $\left.4.33 \mathrm{mmol}\right), \mathrm{FeCl}_{3} \cdot 6 \mathrm{H}_{2} \mathrm{O}(2.34 \mathrm{~g}, 8.66 \mathrm{mmol})$, and $\beta C D$-TDI $(1.5 \mathrm{~g}, 1.30 \mathrm{mmol})$ in $40 \mathrm{~mL}$ of deionised water in a molar ratio of $1: 2: 0.3$, and the reaction mixture was vigorously stirred at a speed of $1200 \mathrm{rpm}$ at $90{ }^{\circ} \mathrm{C}$ for $30 \mathrm{~min}$. Next, in order to generate conglomerated nanoparticles, $5 \mathrm{~mL}$ of $\mathrm{NH}_{4} \mathrm{OH}$ (25\%) was added after the solution was heated to $90{ }^{\circ} \mathrm{C}$, and the reaction mixture was continuously stirred for $1 \mathrm{~h}$ at $90{ }^{\circ} \mathrm{C}$ in an inert atmosphere. In order to remove any unreacted chemicals, the nanoparticles that formed were washed with deionised water five or six times. The resulting product was isolated using an external magnet and was later dried in a vacuum oven at $40{ }^{\circ} \mathrm{C}$.

The modification of the surface of MNP- $\beta C D$-TDI using an IL was adopted from prior works. ${ }^{32,53,54}$ BMIM-Cl $\left(4.0 \mathrm{~g} \mathrm{~L}^{-1}\right)$ was diluted using $25 \mathrm{~mL}$ of absolute ethanol (99.7\%) with the addition of $\mathrm{NaOH}\left(0.01 \mathrm{~mol} \mathrm{~L}^{-1}\right)$ while the $\mathrm{pH}$ of the solution was adjusted to 10.0. After that, MNP- $\beta$ CD-TDI ( $0.5 \mathrm{~g})$ was added to the solution and was stirred at $50{ }^{\circ} \mathrm{C}$ for $30 \mathrm{~min}$ at $280 \mathrm{rpm}$ to allow all the nanoparticles to be suspended in the solution and adhere to the surface of MNP- $\beta C D$-TDI in the presence of the IL. The nanoparticles that formed were then washed with $50 \mathrm{~mL}$ of ACN and deionised water three times. Finally, the resulting product was isolated using an external magnet and was later dried in a vacuum oven at $40{ }^{\circ} \mathrm{C}$. A complete illustration of the synthesised materials is given in Scheme 1.

\subsection{Part 2: Characterisation of adsorbents}

Fourier transform infrared spectroscopy (FT-IR; Thermo Nicolet, Madison, WI, USA) was carried out in the wavenumber range between $4000 \mathrm{~cm}^{-1}$ and $400 \mathrm{~cm}^{-1}$. Moreover, carbonhydrogen-nitrogen analysis (PerkinElmer 2400 Series II CHN analyser, Massachusetts, USA) was performed to determine the amounts of $\mathrm{C}, \mathrm{H}$, and $\mathrm{N}$ present. Next, the magnetisations of the bare and both modified MNPs were measured using a vibrating sample magnetometer (Lake Shore 7404 series, McCorkle
Boulevard, Westerville, OH, USA). The morphology and particle size, on the other hand, were determined using a scanning electron microscope (Quanta FEG650, Oxford Instruments, Hillsboro, USA) and a transmission electron microscope (FEI CM12, Hillsboro, USA). After that, a surface area analyser was employed to determine the surface area and porous properties of the materials via nitrogen adsorption-desorption analysis at $77 \mathrm{~K}$ using the Brunauer-Emmett-Teller (BET) method (Quantachrome, Boynton Beach, FL, USA). The thermal stability of the synthesised materials was later investigated by performing thermogravimetric analysis (TGA; PerkinElmer TGA-STA 1500, Massachusetts, USA) at a heating rate of $10{ }^{\circ} \mathrm{C} \mathrm{min}^{-1}$ between 30 and $900{ }^{\circ} \mathrm{C}$ under a nitrogen atmosphere. X-ray diffraction patterns were recorded using an Empyrean X-ray diffractometer (PANalytical, Almelo, Netherlands) from $2 \theta=10^{\circ}$ to $90^{\circ}$ with $\mathrm{Cu}$ $\mathrm{K} \alpha$ radiation $(\lambda=1.5418 \AA)$ at a scan rate of $0.02^{\circ} \mathrm{s}^{-1}$. Finally, nuclear magnetic resonance (NMR, Bruker Avance III $700 \mathrm{MHz}$, Rheinstetten, Ettlingen, Germany) was employed to characterise the formation of the inclusion complex of $\beta C D$ with ArP.

\subsection{Part 3: Batch removal experiments}

2.4.1 Preliminary batch study. The prepared adsorbents were examined in adsorption studies in order to compare the performance of IL-MNP- $\beta C D$-TDI with that of MNP- $\beta C D$-TDI and native MNPs. The removal conditions were: temperature, $25{ }^{\circ} \mathrm{C}$; analyte solution, $10 \mathrm{~mL}$; sorbent dosage, $20 \mathrm{mg}$; equilibrium time, $2 \mathrm{~h}$ at $250 \mathrm{rpm}$.

2.4.2 Sorption experiments. PP, BP, and ArP were selected as analytes for batch adsorption experiments, and several parameters, such as the effect of $\mathrm{pH}$, kinetics, effect of contact time, effect of concentration, sorbent dosage, and reusability, were optimised by employing a batch method using $20 \mathrm{mg}$ of ILMNP- $\beta C D$-TDI, $10 \mathrm{~mL}$ of an aqueous solution that contained the analytes of interest at a known concentration $\left(10 \mathrm{mg} \mathrm{L}^{-1}\right)$ in a tightly sealed vial, and a shaking time of $2 \mathrm{~h}(180 \mathrm{rpm})$ at room temperature. After the adsorption process, the adsorbent was separated using an external magnetic field and was filtered prior to analysis with an ultraviolet-visible spectrometer (PerkinElmer Lambda 25, Massachusetts, USA) equipped with $10 \mathrm{~mm}$ quartz cuvettes (PerkinElmer, Massachusetts, USA) at wavelengths of $256 \mathrm{~nm}$ (PP and BP) and $257 \mathrm{~nm}$ (ArP). All the samples were tested in triplicate, whereas the removal efficiency $(R \%)$ was calculated using the following equation:

$$
R \%=\frac{\left(C_{\mathrm{o}}-C_{\mathrm{e}}\right)}{C_{\mathrm{o}}} \times 100
$$

The amount of the analytes adsorbed per unit mass of the adsorbent $\left(q_{\mathrm{e}}\right)$ was calculated as follows:

$$
q_{\mathrm{e}}=\frac{\left(C_{\mathrm{o}}-C_{\mathrm{e}}\right) V}{W}
$$

where $C_{\mathrm{o}}$ and $C_{\mathrm{e}}$ are the initial and equilibrium concentrations of the solution $\left(\mathrm{mg} \mathrm{L}^{-1}\right)$, respectively, $V(\mathrm{~L})$ denotes the volume of the solution, whereas $W(\mathrm{~g})$ refers to the mass of the dry adsorbent used. 

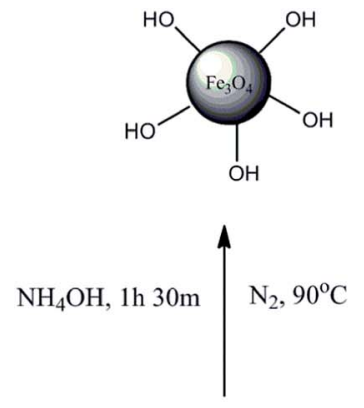

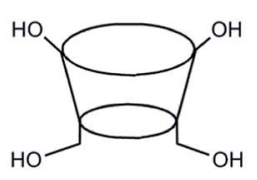

$\mathrm{DMF}, 24 \mathrm{~h}, 70^{\circ} \mathrm{C}$

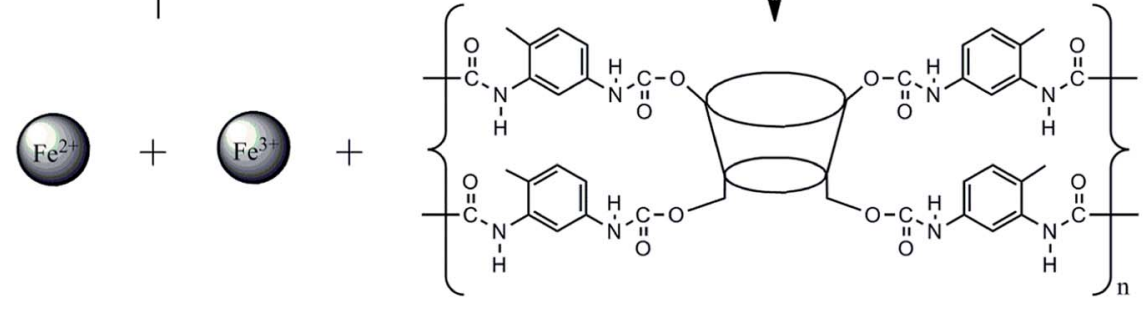

$\mathrm{NH}_{4} \mathrm{OH}, 1 \mathrm{~h} 30 \mathrm{~m}$ $\mathrm{N}_{2}, 90^{\circ} \mathrm{C}$

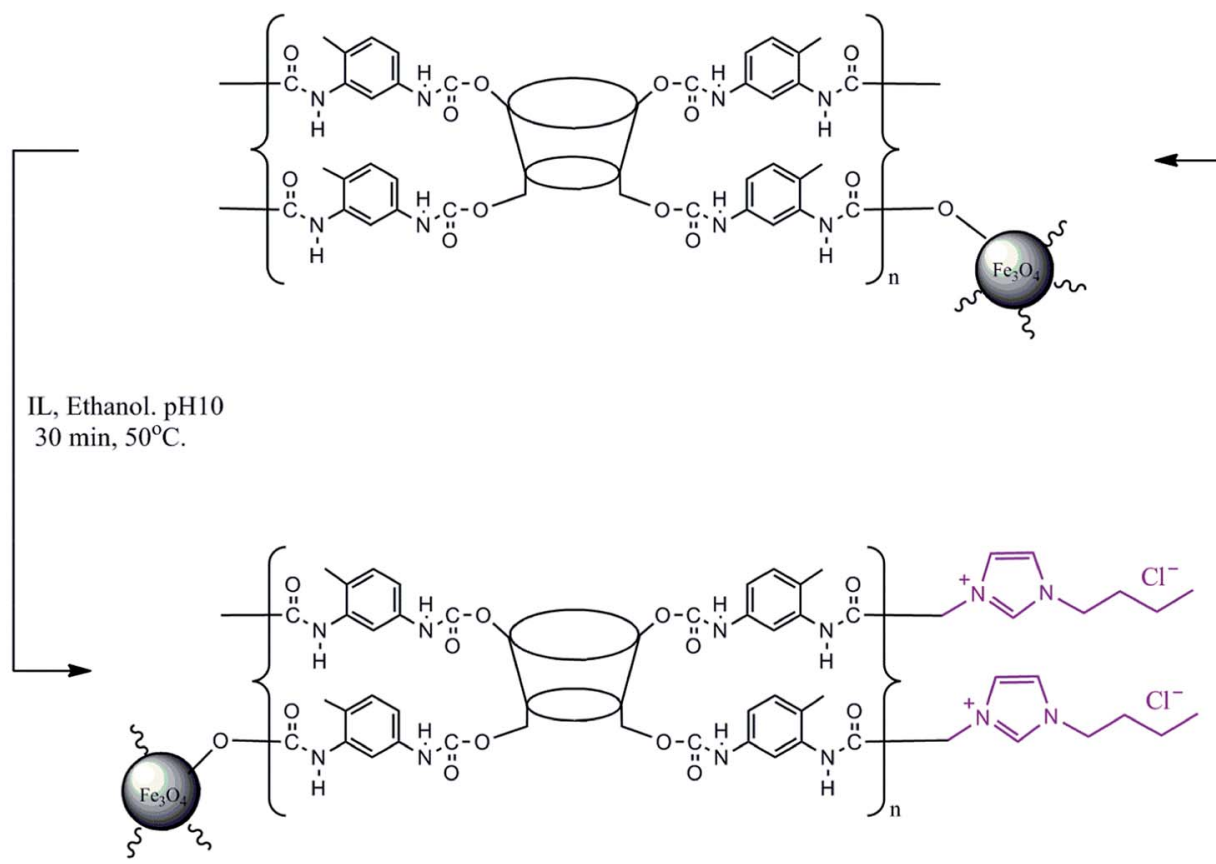

Scheme 1 Complete illustration of the synthesised materials.

2.4.3 Effect of solution $\mathbf{p H}$. Adsorption was performed at various $\mathrm{pH}$ values that ranged from 3 to 10 at room temperature. The desired $\mathrm{pH}$ was adjusted with $0.01 \mathrm{M} \mathrm{HCl}$ and $0.01 \mathrm{M} \mathrm{NaOH}$ using a pH meter (OHAUS Starter 3100, Ohio, USA). The initial concentrations of the analytes were fixed at $10 \mathrm{mg} \mathrm{L}^{-1}$ in $10 \mathrm{~mL}$ of an aqueous solution of the analytes with a sorbent dosage of $20 \mathrm{mg}$, which was agitated at $250 \mathrm{rpm}$ for $120 \mathrm{~min}$.

2.4.4 Effect of contact time. The effect of the contact time on the removal of parabens using IL-MNP- $\beta C D$-TDI was investigated at various time intervals of between 0 and $120 \mathrm{~min}$ at room temperature. The initial concentrations of the analytes were fixed at $10 \mathrm{mg} \mathrm{L}^{-1}$ in $10 \mathrm{~mL}$ of an aqueous solution of the analytes with a sorbent dosage of $20 \mathrm{mg}$ at a $\mathrm{pH}$ of 6 for all the studied analytes, and the solution was agitated at $250 \mathrm{rpm}$.

2.4.5 Effect of initial concentration. Equilibrium studies were performed at various initial concentrations of the analytes within the range of $0-100 \mathrm{mg} \mathrm{L}^{-1}$ in $10 \mathrm{~mL}$ of an aqueous solution of the analytes with a sorbent dosage of $20 \mathrm{mg}$ at a $\mathrm{pH}$ of 6 at three different temperatures, namely, $298 \mathrm{~K}, 318 \mathrm{~K}$, and $338 \mathrm{~K}$, and the solution was later agitated at $250 \mathrm{rpm}$ for $80 \mathrm{~min}$.

2.4.6 Effect of sorbent dosage. The effect of the sorbent (ILMNP- $\beta C D$-TDI) dosage on the adsorption process was determined by varying the sorbent dosage from 20 to $120 \mathrm{mg}$ with an analyte concentration of $80 \mathrm{mg} \mathrm{L}^{-1}$ at a $\mathrm{pH}$ of 6 and a temperature of $298 \mathrm{~K}$. The appropriate sorbent dosage of IL-MNP- $\beta \mathrm{CD}$ - 
TDI was weighed under a tightly sealed vial, and the solution was agitated at $250 \mathrm{rpm}$ for $80 \mathrm{~min}$. The adsorption capacity ( $\mathrm{mg}$ $\mathrm{g}^{-1}$ ) per unit mass was calculated using eqn (2).

2.4.7 Preparation of real samples. Three types of water samples, namely, tap water, drain water, and industrial wastewater, were collected and filtered using $0.45 \mu \mathrm{m}$ membrane filters and were then stored in the dark at $4{ }^{\circ} \mathrm{C}$. After that, the samples were spiked at a concentration of $80 \mathrm{mg} \mathrm{L}^{-1}$ in $10 \mathrm{~mL}$ and were directly added to a vial that contained $100 \mathrm{mg}$ of the sorbent for a further adsorption process. Batch experiments were conducted in triplicate $(n=3)$ so as to ensure the accuracy and precision of the results.

2.4.8 Regeneration of IL-MNP- $\boldsymbol{\beta C D}$-TDI adsorbent. The reusability of IL-MNP- $\beta$ CD-TDI was determined five times via adsorption-desorption cycles. Firstly, after the adsorption process, the wet sorbent was collected and immersed in $5 \mathrm{~mL}$ of deionised water, which was subsequently vortexed for $1 \mathrm{~min}$, and the sorbent was isolated using an external magnet. A similar procedure was repeated by replacing the deionised water with ACN, and the sorbent was then dried for $1 \mathrm{~h}$ in a vacuum oven.

2.4.9 Synthesis and characterization of $\boldsymbol{\beta C D}-\mathbf{A r P}$ inclusion complex. The inclusion complex was prepared via a conventional kneading method. ${ }^{55}$ Equimolar amounts of $\beta C D$ and ArP were kneaded for approximately $30 \mathrm{~min}$ with a mortar and pestle in a minimal amount of analytical-grade absolute ethanol (denatured, 99.7\%), which was purchased from J. Kollin
Chemicals (Midlothian, UK), to produce a homogeneous paste and dried to a constant mass. After drying, a white powdery complex was obtained and characterized using ${ }^{1} \mathrm{H}$ NMR. The calculated yield was approximately $65.8 \%$.

2.4.10 Preparation of $\boldsymbol{\beta C D}$-ArP for spectroscopic studies. A $2 \mathrm{~mL}$ portion of $0.01 \mathrm{mM}$ ArP, $3.2 \mathrm{~mL}$ of a $0.004 \mathrm{M}$ solution of $\beta C D$, and $2 \mathrm{~mL}$ of a buffer solution $(\mathrm{pH} 7)$ were transferred accurately to a $10 \mathrm{~mL}$ volumetric flask and diluted to the mark using deionised water, which was then mixed well and agitated for 5 min via ultrasound. After that, the solution was left for $30 \mathrm{~min}$ at room temperature. The absorption spectrum of the $\beta C D-A r P$ complex was recorded against a blank reagent, which was prepared with the same reagent concentration but in the absence of ArP. Furthermore, the absorption spectra of $\beta C D$ and ArP were recorded in accordance with the same procedure. All absorbances were measured at $257 \mathrm{~nm}$ against the blank reagent. Moreover, to generate a constant curve, the concentration of ArP was kept constant at $0.01 \mathrm{mM}$ but the concentration of $\beta C D$ was varied $(0.004,0.005,0.007,0.009$, and 0.01 $\mathrm{M})$. This procedure was repeated so as to obtain three or more absorbance values for each $\beta C D$ concentration that was studied.

\section{Results and discussion}

\subsection{Characterisation of materials}

The results of the FT-IR and TGA analyses and the BET hysteresis loops in this study are illustrated in Fig. 1, whereas Table 1
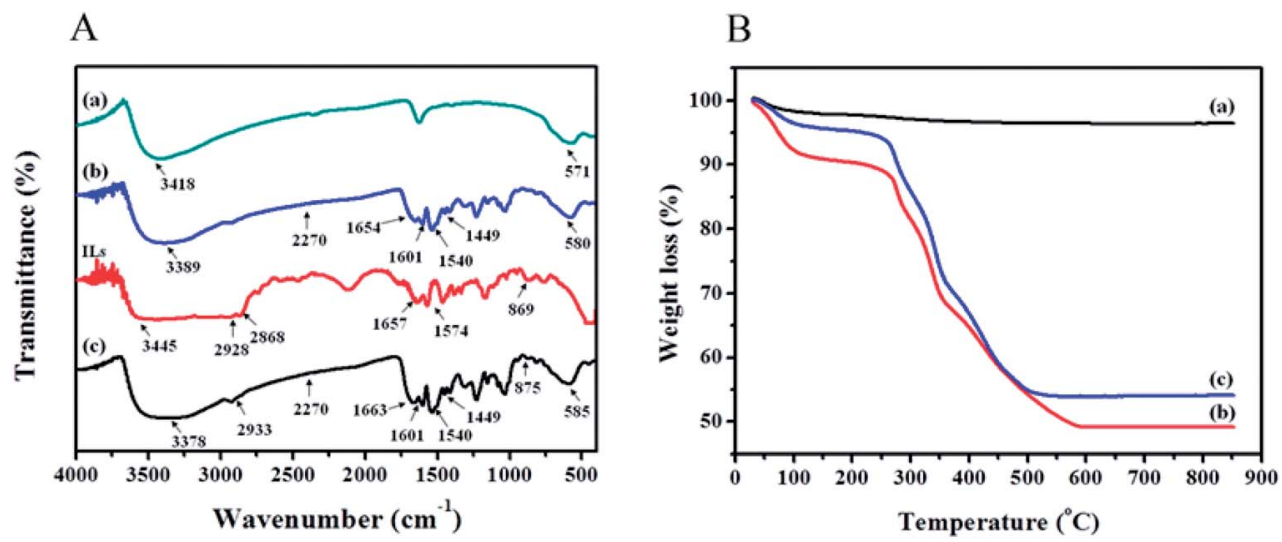

$\mathrm{C}$
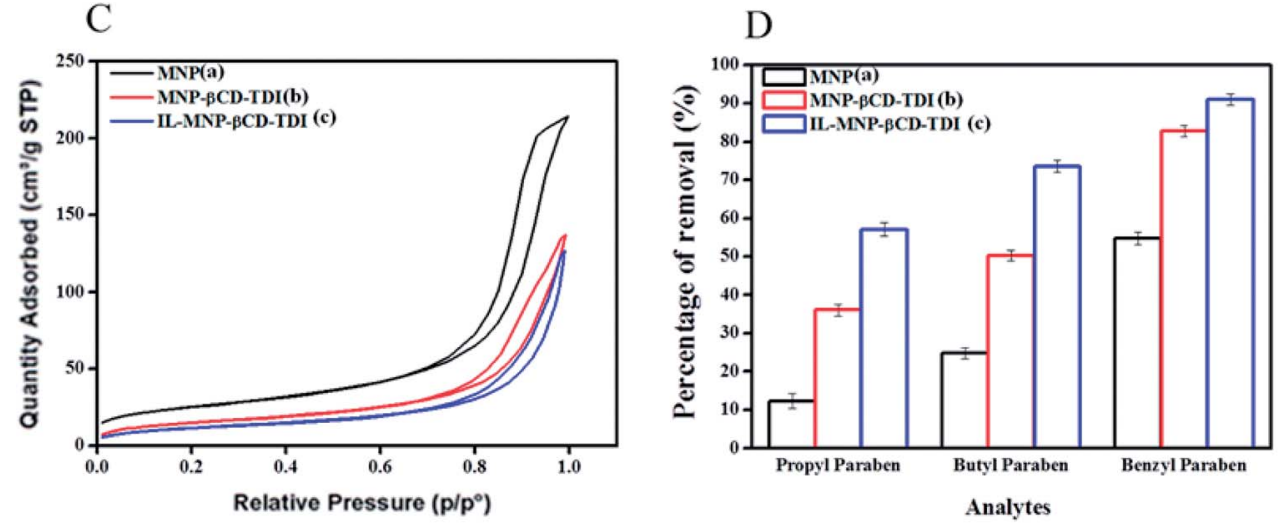

Fig. 1 (A-C) Present the results of the characterisation of the synthesised adsorbents, whereas (D) shows the results of preliminary batch adsorption experiments for comparison between (a) MNPs, (b) MNP- $\beta C D-T D I$, and (c) IL-MNP- $\beta C D-T D I$. 
Table 1 Physicochemical properties determined by FT-IR, TGA, BET and CHN analyses

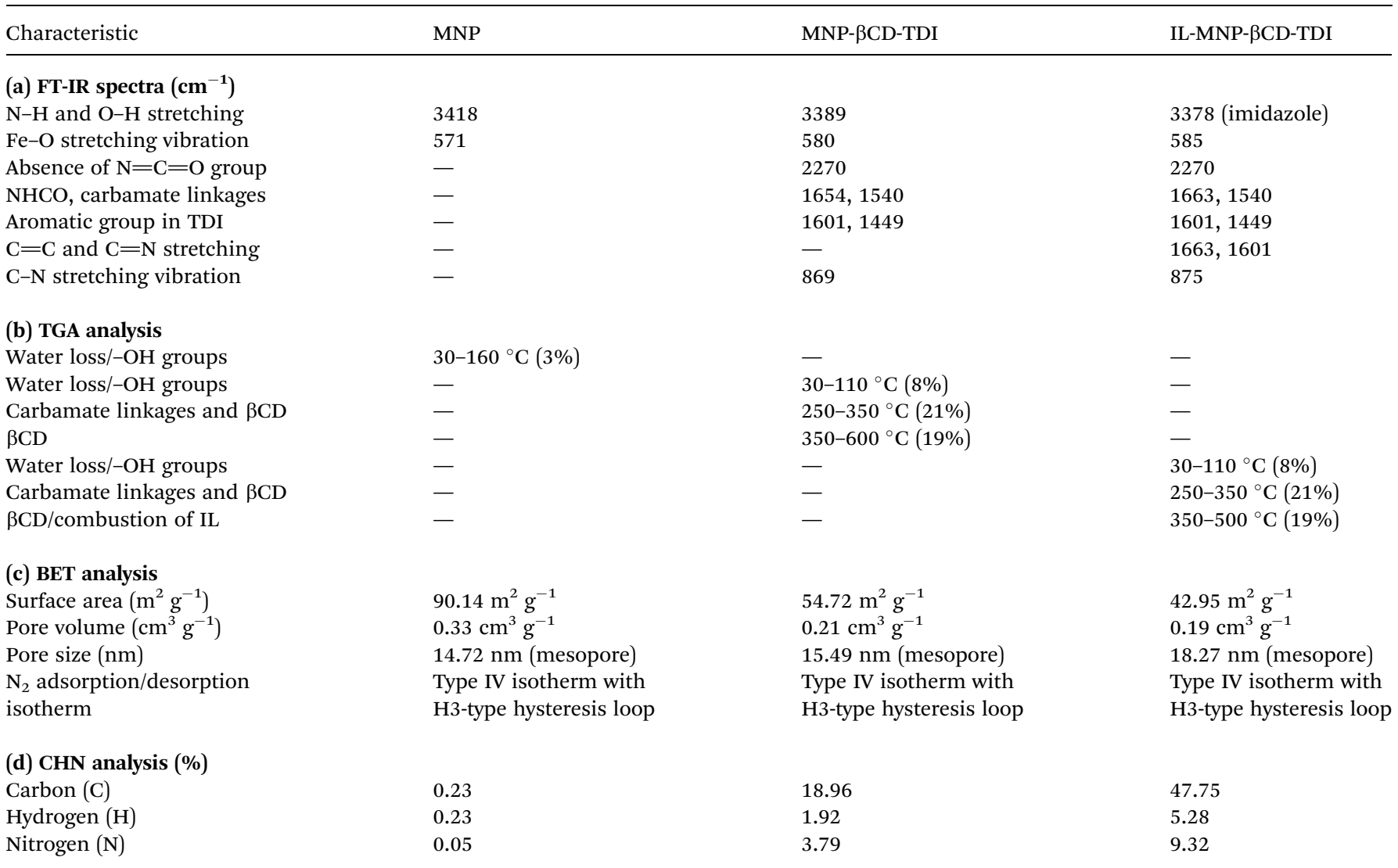

summarises the results of the (a) FT-IR, (b) TGA, (c) BET and (d) CHN analyses in detail. The FT-IR spectra (Fig. 1A) of MNPs, MNP- $\beta$ CD-TDI, IL (BMIM-Cl), and IL-MNP- $\beta$ CD-TDI were recorded using the $\mathrm{KBr}$ method. As a result, the presence of magnetic properties in the MNPs corresponding to curves (a), (b), and (c) is proven by the appearance of the $\mathrm{Fe}-\mathrm{O}$ peak, which represents the tetrahedral form of pure MNPs, at about 570$590 \mathrm{~cm}^{-1}$. Furthermore, the absence of a peak at $2270 \mathrm{~cm}^{-1}$ (corresponding to the isocyanate group) in curves (b) and (c) indicates the success of the polymerisation process, together with the presence of a peak due to carbamate (NHCOO) linkages at $1654 \mathrm{~cm}^{-1} .{ }^{56}$ In the case of $\mathrm{Fe}_{3} \mathrm{O}_{4}$, the broad absorption peak at around $3400 \mathrm{~cm}^{-1}$ signifies the presence of surface hydroxyl groups (O-H stretching), whereas the broad -OH stretching band of MNP- $\beta C D$-TDI at about $3300-3400 \mathrm{~cm}^{-1}$ is attributable to multiple $-\mathrm{OH}$ functional groups and appears to decrease owing to cross-linking with TDI. Moreover, as for the FT-IR spectrum of IL-MNP- $\beta$ CD-TDI in curve (d), the peak broadening observed at $2900-3400 \mathrm{~cm}^{-1}$ indicates the successful loading of the IL onto MNP- $\beta$ CD-TDI.

VSM and XRD analyses were reported in a prior work ${ }^{51}$ in which the magnetic behaviour of the synthesised adsorbent was determined via VSM analysis. In order to separate the MNPs from the solution, a magnetisation (Ms) of $16.3 \mathrm{emu} \mathrm{g}^{-1}$ seemed scientifically adequate because a higher Ms value facilitates the separation of MNPs from the solution. ${ }^{57}$ The maximum Ms value of the MNPs in this study was $60.6 \mathrm{emu}^{-1}$ (a), but in the presence of the polymeric materials coated on the surface of the MNPs the value of Ms decreased to 46.8 emu g ${ }^{-1}$ (b). ${ }^{4,58}$ Upon loading with the IL, the value of Ms appeared to be lower than that of the first coating, namely, $25.0 \mathrm{emu}^{-1}$ (c). This confirms the successful coating of the IL onto the surface of MNP- $\beta C D$ TDI.

The crystal structures of all the synthesised adsorbents were successfully analysed via XRD. The characteristic diffraction peaks of the MNPs appeared to correspond to those of standard $\mathrm{Fe}_{3} \mathrm{O}_{4}$ crystals in accordance with JCPDS card number 19-0629. Diffraction peaks of the MNPs were observed at $2 \theta=30.44^{\circ}$, $35.74^{\circ}, 43.54^{\circ}, 53.81^{\circ}, 57.50^{\circ}$, and $63.04^{\circ}$, which corresponded to the (220), (311), (400), (422), (511), and (440) cubic spinel planes of $\mathrm{Fe}_{3} \mathrm{O}_{4}$, respectively. The characteristic peaks of MNP$\beta C D-T D I$ and IL-MNP- $\beta C D$-TDI, however, did not differ from the peaks of the MNPs, which thus confirmed that alteration of the surface of the MNPs did not affect their crystal phase. Furthermore, in terms of intensity, the decrease for MNP- $\beta C D$ TDI and the increase for IL-MNP- $\beta$ CD-TDI are attributable to the presence of an amorphous layer of the $\beta C D$ polymer, as well as the successful coating of the IL onto the surface of MNP- $\beta C D$ TDI. ${ }^{58}$

The thermal properties (Fig. 1B) of the MNPs were determined from the TGA curves, which indicated a three-stage degradation pattern. Basically, the first step of the degradation process (MNPs) corresponds to the loss of water at lower temperatures $\left(30-160{ }^{\circ} \mathrm{C}\right)$. Next, the second stage of degradation 
involved both MNP- $\beta C D$-TDI and IL-MNP- $\beta C D$-TDI owing to the presence of carbamate linkages and $\beta C D\left(250-350{ }^{\circ} \mathrm{C}\right)$, with a weight loss of $21 \%$. The low weight loss shows that these adsorbents were thermally stable at high temperatures and the transformation of $\beta C D$ into a polymeric form had increased its stability. ${ }^{\mathbf{3 8 5 9}}$ The last stage of the degradation process occurred in the range of $350-600{ }^{\circ} \mathrm{C}$ and was perhaps due to the thermal decomposition of $\mathrm{CD}$, which thus confirmed the grafting of the $\beta C D$ polymer onto the surface of MNPs, with a weight loss of $19 \%$ for both materials. Apart from that, the weight loss (350$500{ }^{\circ} \mathrm{C}$ ) for IL-MNP- $\beta$ CD-TDI appears to be a result of thermal carbonisation and decomposition of organic groups, such as combustion of the IL. ${ }^{58}$ The trends in degradation and weight loss are detailed in Table 1(b).

The results of the SEM and TEM analyses are illustrated in Fig. S1 (ESI S1 $\uparrow$ ). The SEM analysis was performed to determine the surface morphologies of the adsorbents. The micrograph that was obtained displayed several similarities in the morphologies because the adsorbents were generally synthesised from the same base, which was magnetic. Fig. S1(a) $\dagger$ shows the surface morphology of the synthesised MNPs, whereas Fig. S1(b) $\dagger$ shows the chemical coating of the $\beta C D$ polymer onto the surface of MNPs. The polymerization process with TDI led to a morphology that resembled a conglomerate of beads with small cavities surrounding the MNPs. Furthermore, upon the loading of the IL, as shown in Fig. S1(c), $\uparrow$ the presence of the IL is clearly indicated on the surface of MNP- $\beta C D$-TDI (whitish appearance).

TEM was performed for two primary purposes: (1) to exemplify the core-shell structure of the adsorbents and (2) to confirm the spherical morphology of the agglomerates, as illustrated in Fig. S1 $\left(a^{\prime}\right)-\left(c^{\prime}\right)$. $\uparrow$ The average diameter of the bare MNPs was approximately $14 \mathrm{~nm}$ but increased slightly to $16 \mathrm{~nm}$ after coating with the $\beta C D$ polymer. Subsequently, the presence of the IL on the surface of MNP- $\beta C D$-TDI resulted in welldispersed absorbance and an increase in the particle diameter to $18 \mathrm{~nm}$. Initially, the TEM images were analysed with ImageJ software in order to determine the average diameter from the particle distribution of the adsorbents. This step was carried out prior to the BET analysis so as to ascertain the accuracy of the analysis classification (microporous, mesoporous or macroporous). On the basis of the tabulated histogram data, the average diameter of the particles appeared to be between $2 \mathrm{~nm}$ and $6 \mathrm{~nm}$, which corresponded to the mesoporous category (2$50 \mathrm{~nm})$.

Next, Table 1(c) presents the findings obtained from the BET analyses that were performed for all the adsorbents. The surface area of the bare MNPs was $90.14 \mathrm{~m}^{2} \mathrm{~g}^{-1}$ and decreased to 54.72 $\mathrm{m}^{2} \mathrm{~g}^{-1}$ after they were coated with the polymeric adsorbent. Upon the introduction of the IL, the surface area of the adsorbent decreased to $42.95 \mathrm{~m}^{2} \mathrm{~g}^{-1}$, which appeared to be an effect of the coating of the MNPs. In addition, the Barrett-JoynerHalenda (BJH) model was employed in this study to calculate the pore size distribution of the adsorbents. The average pore sizes of these adsorbents were $14.72 \mathrm{~nm}$ for MNPs, $15.49 \mathrm{~nm}$ for MNP- $\beta C D$-TDI, and up to $18.27 \mathrm{~nm}$ upon the loading of the IL, which hence indicated the mesoporous type of adsorbent owing to a decrease in pore volume from $0.33 \mathrm{~cm}^{3} \mathrm{~g}^{-1}$ to $0.21 \mathrm{~cm}^{3} \mathrm{~g}^{-1}$ and $0.19 \mathrm{~cm}^{3} \mathrm{~g}^{-1}$, respectively. Such findings, nevertheless, appear to contradict those of a prior study, in which MNPs coated with $\beta C D$ and functionalized with an IL for a sensor application displayed increases in both surface area and pore volume. ${ }^{52}$ Such a contrast may be attributed to the unique properties of $\mathrm{CD}$ and the IL. Furthermore, the $\mathrm{N}_{2}$ adsorption/ desorption isotherms showed that all the adsorbents exhibited typical type IV isotherms, which correspond to the mesoporous type of material, with H3-type hysteresis loops, which relate to non-rigid aggregates of plate-like particles, as portrayed in Fig. 1C. ${ }^{58}$

Next, the results obtained from the CHN analyses are tabulated in Table 1(d). The elemental compositions of the adsorbents were determined by a CHN analyser. As a result, the $\mathrm{CHN}$ findings confirmed the successful coating of the $\beta C D$ polymer on the surface of the MNPs, with increases in the following contents: $18.96 \%$ of $\mathrm{C}, 1.92 \%$ of $\mathrm{H}$, and $3.79 \%$ of $\mathrm{N}$ from the low amounts in the MNPs, which were $0.23 \%$ of both $\mathrm{C}$ and $\mathrm{H}$ and $0.05 \%$ of $\mathrm{N}$. As indicated in the table, the nitrogen content rose to $9.32 \%$ owing to the presence of the IL. ${ }^{60}$ This demonstrates that the loading of the IL onto the surface of MNP- $\beta C D$-TDI was indeed a success, together with increases in elemental percentages to $47.75 \%$ of $\mathrm{C}$ and $5.28 \%$ of $\mathrm{H}$.

\subsection{Optimisation of batch experiments}

3.2.1 Preliminary batch study. The results presented in Fig. 1D show the efficiency of the adsorption of the three selected parabens (PP, BP, and ArP) by the synthesised materials. As the primary focus of this study is on the newly developed materials, the results appear to indicate a significant increase in adsorption efficiency for (c) in comparison with (a) and (b). Moreover, the presence of the IL in (c) greatly increased the selectivity towards parabens owing to various factors, such as hydrophobic interactions between $\beta \mathrm{CD}$ and those parabens, including $\pi-\pi$ interactions and hydrogen bonding between the aromatic rings in the parabens and the imidazolium ring in ILMNP- $\beta C D$-TDI. In addition, the performance of this material was further investigated and the formation of the inclusion complex between $\beta \mathrm{CD}$ and one selected paraben (ArP) was analysed.

3.2.2 Effect of solution $\mathbf{p H}$. The effect of $\mathrm{pH}$ values that ranged from 3 to 10 on the adsorption efficiency of the three types of adsorbents for the selected parabens (PP, BP, and ArP) is illustrated in Fig. 2. The findings indicate that the sorption was $\mathrm{pH}$-dependent and a $\mathrm{pH}$ of 6 was optimal for all the studied parabens. The optimum $\mathrm{pH}$ determined from the graphs indicates the adsorption efficiency of the MNPs, which was $19.10 \%$, $32.22 \%$, and $70.80 \%$ for PP, BP, and ArP, respectively, whereas the values for MNP- $\beta$ CD-TDI exhibited an increase as follows: $50.19 \%, 63.85 \%$, and $84.76 \%$, respectively. Upon the loading of the IL, the percentage removal for IL-MNP- $\beta$ CD-TDI significantly increased to $60.37 \%, 79.31 \%$, and $91.69 \%$ for PP, BP, and ArP, respectively, which was mainly due to the presence of the IL, which enhanced the hydrophobicity of the polymer. ${ }^{40}$ Besides, this could also be due to the presence of more active 

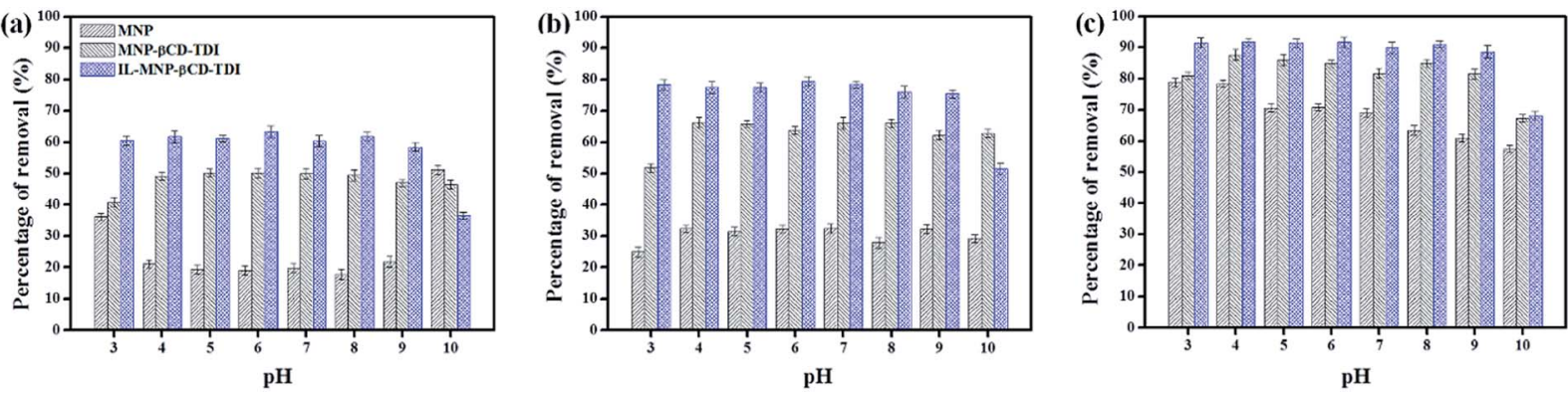

Fig. 2 Effect of initial pH on the adsorption of (a) PP, (b) BP, and (c) ArP using three different types of materials (conditions: sorbent, 20 mg; initial concentration, $10 \mathrm{mg} \mathrm{L}^{-1}$; adsorption time, $120 \mathrm{~min}$; temperature, $298 \mathrm{~K}$ ).

sites in IL-MNP-BCD-TDI, as well as the physical interactions between the adsorbent and the adsorbates dominated by van der Waals forces, formation of inclusion complexes, and hydrogen bonding..$^{29,37}$ In this study, the formation of inclusion complexes seems to emerge as the main interaction, because the cavity of $\beta C D$ was retained throughout polymerisation and coating of the polymer onto the surface of MNPs. Moreover, the $\pi-\pi$ interaction between the imidazolium ring in the $\mathrm{IL}$ and all the studied parabens may also be a reason for the higher removal efficiency of IL-MNP- $\beta$ CD-TDI.

After comparing the types of adsorbents, IL-MNP- $\beta$ CD-TDI was selected as the adsorbent for this study. As evidenced in Fig. 2, the adsorption efficiency displayed by IL-MNP-BCD-TDI increased but appeared to exhibit slightly similar trends for all the studied parabens (PP, $\mathrm{BP}$, and $\mathrm{ArP}$ ) from a $\mathrm{pH}$ of 3 to a $\mathrm{pH}$ of 6. The optimum $\mathrm{pH}$ refers to a $\mathrm{pH}$ of 6 , and a decline in adsorption efficiency was observed from a pH of 7 to a pH of 10 . In acidic conditions $(\mathrm{pH}<6)$, the amount of protons was maximized owing to protonation of the remaining hydroxyl groups on the surface of MNPs, which may saturate the sorbent sites and thus generate a more cationic surface of IL-MNP- $\beta C D-$ TDI. ${ }^{4,58}$ In addition, the parabens were also in their protonated form as they only participate in $\pi-\pi$ interaction and electrostatic repulsion. At a $\mathrm{pH}$ of 6 , nonetheless, the paraben compounds began to adopt their neutral forms, which hence facilitated their participation in hydrophobic interactions with the $\beta \mathrm{CD}$ polymer cavity, whereas the presence of imidazole rings in the IL suggests $\pi-\pi$ interactions and hydrogen bonding. In basic conditions $(\mathrm{pH}>6)$, deprotonation of residual hydroxyl groups on MNPs could make the surface of the adsorbent anionic and hence decrease the hydrophobicity of the adsorbent. Because the $\mathrm{p} K_{\mathrm{a}}$ values of the parabens are approximately 8.3, they also exist in the anionic form, which thus results in fewer interactions between the targeted analyte and the adsorbent. In brief, as the formation of an inclusion complex is deemed to be impossible with protonated and deprotonated parabens, a $\mathrm{pH}$ of 6 appears to be favourable because the parabens are neutral at this $\mathrm{pH}$ and can form inclusion complexes with the $\beta \mathrm{CD}$ cavity.

3.2.3 Effect of contact time. The effect of the contact time on the removal of the parabens (PP, BP, and ArP) by IL-MNP$\beta C D$-TDI was thoroughly investigated within the time range of 0-120 min at room temperature $(298 \mathrm{~K})$, as illustrated in Fig. 3.
The adsorption efficiency for the studied parabens seemed to be rapid during the first $5 \mathrm{~min}(\mathrm{ArP}), 20 \mathrm{~min}(\mathrm{BP})$, and $40 \mathrm{~min}(\mathrm{PP})$, owing to the availability of additional active sites on the surface of IL-MNP- $\beta C D$-TDI. Nevertheless, the time taken for the parabens to interact with these active sites strongly depended on the alkyl chains present within the parabens. Therefore, the longer was the alkyl chain ( $\mathrm{ArP}>\mathrm{BP}>\mathrm{PP}$ ), the longer was the time taken by the parabens to saturate the active sites. Besides, the adsorption process was carried out up to $120 \mathrm{~min}$, whereas the optimum contact time for all the studied parabens was $80 \mathrm{~min}$, with efficiencies of $67.03 \%, 80.33 \%$, and $92.61 \%$ for PP, BP, and ArP, respectively. After $80 \mathrm{~min}$, the adsorption rate was observed to be constant. Hence, $80 \mathrm{~min}$ was selected for all the studied parabens as the equilibrium point throughout the study.

3.2.4 Effect of initial concentration. Adsorption experiments at initial paraben concentrations of 5 to $120 \mathrm{mg} \mathrm{L}^{-1}$ were performed by maintaining the amount of the adsorbent (ILMNP- $\beta C D$-TDI) at $20 \mathrm{mg}$ at the optimum $\mathrm{pH}$ for $80 \mathrm{~min}$ at various temperatures, namely, $298 \mathrm{~K}, 318 \mathrm{~K}$, and $338 \mathrm{~K}$, for which the results are presented in Fig. 4A. The findings indicate that the percentage removal for all the studied parabens ( $\mathrm{ArP}>$ $\mathrm{BP}>\mathrm{PP}$ ) decreased with an increase in the initial concentration. This can be explained by the fact that the surface of the adsorbent has a limited number of active sites, which suggests

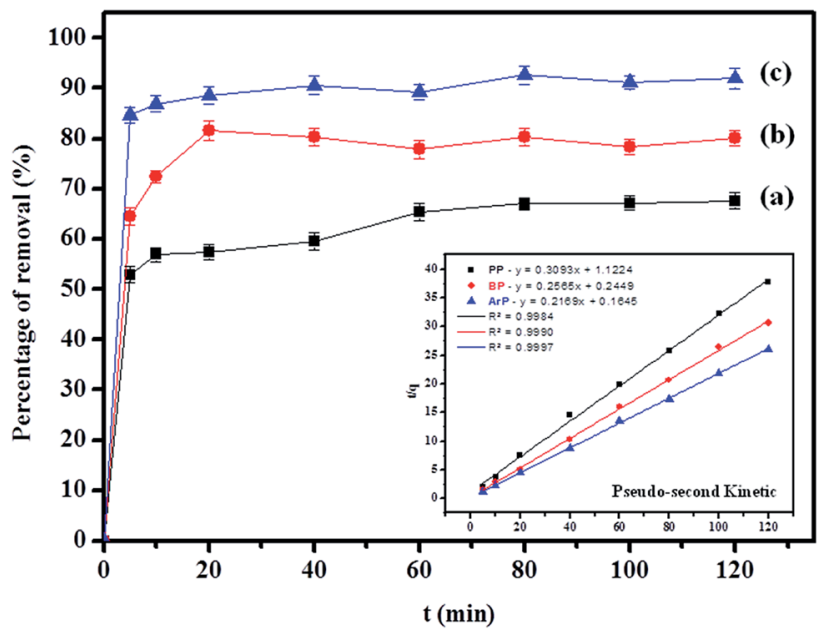

Fig. 3 Effect of contact time on removal of (a) PP, (b) BP, and (c) ArP using IL-MNP- $\beta C D$-TDI (conditions: sorbent, $20 \mathrm{mg}$; initial concentration, $10 \mathrm{mg} \mathrm{L}^{-1}$; sample $\mathrm{pH}$, 6; temperature, $298 \mathrm{~K}$ ). 

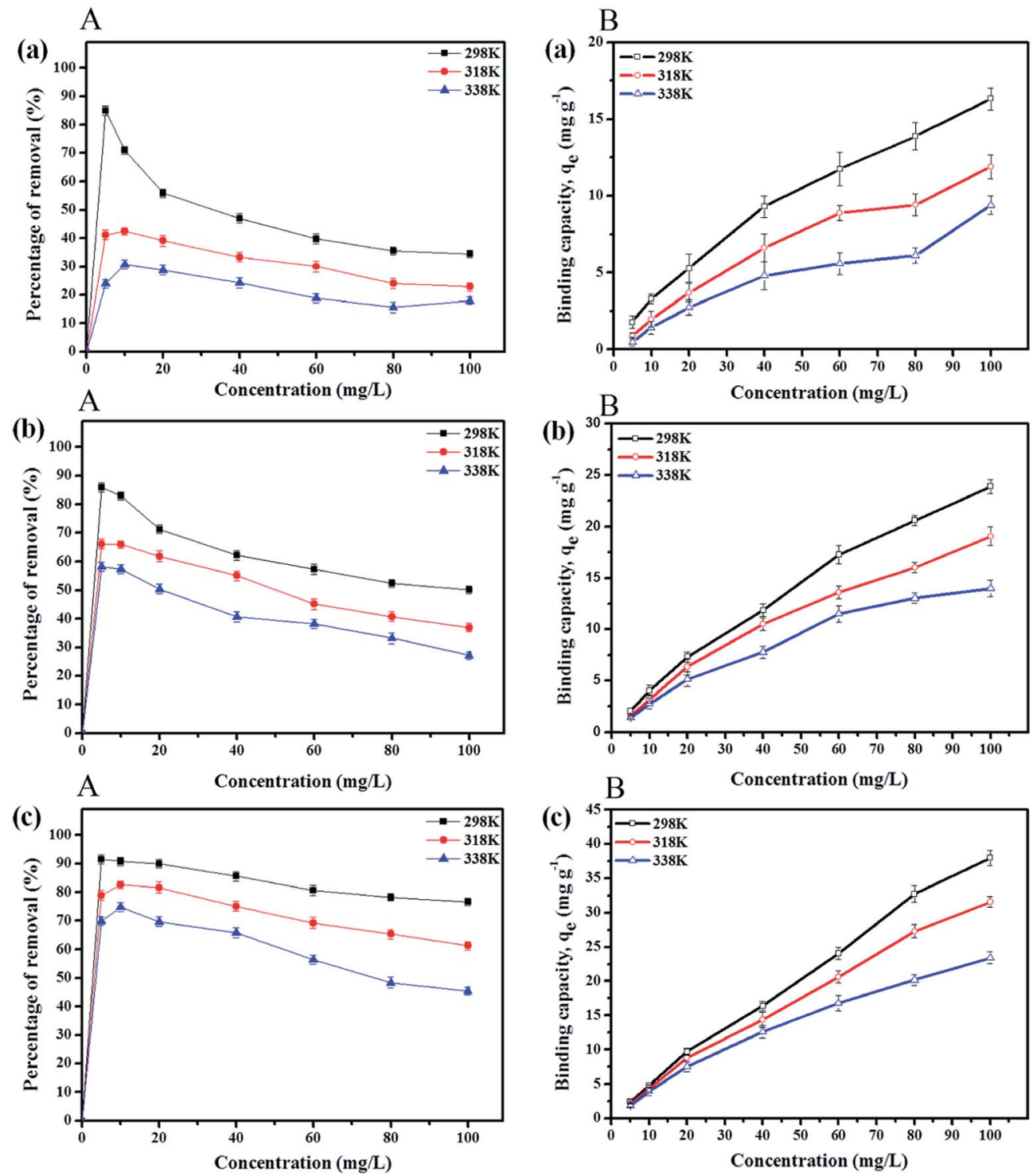

Fig. 4 Effects of initial concentration (A) and solution temperature (B) on removal of (a) PP, (b) BP, and (c) ArP using IL-MNP- $\beta C D-T D I$ at various temperatures (conditions: sorbent, 20 mg; adsorption time, $80 \mathrm{~min}$; sample $\mathrm{pH}, 6$ ).

that saturation of the paraben compounds occurs above certain concentrations. Besides, the temperature also greatly affected the removal of all the studied parabens, as a rise in temperature reduced the rate of removal, which was perhaps due to degradation of the parabens at higher temperatures. Thus, the percentage removal reached equilibrium at $80 \mathrm{mg} \mathrm{L}^{-1}$ at room temperature ( $298 \mathrm{~K})$.

3.2.5 Effect of solution temperature. The temperature also influenced the adsorption capacities $q_{\mathrm{e}}\left(\mathrm{mg} \mathrm{g}^{-1}\right)$ for the paraben compounds (PP, BP, and ArP), as illustrated in Fig. 4B. The graph portrays the trends displayed in the adsorption capacity for all the studied parabens, which seemed to decrease with an increase in temperature. Moreover, insignificant variations were discovered in the adsorption capacities at all the studied temperatures $(298 \mathrm{~K}, 318 \mathrm{~K}$, and $338 \mathrm{~K}$ ) at lower concentrations (5 and $10 \mathrm{mg} \mathrm{L}^{-1}$ ). Nevertheless, the adsorption capacities appeared to be optimal at room temperature (298 K) at higher concentrations ( 80 and $100 \mathrm{mg} \mathrm{L}^{-1}$ ). These results indicate the exothermic nature of the adsorption process for all the studied parabens, which was largely due to the tendency of the parabens towards desorption from the surface of the adsorbent (IL-MNP$\beta C D$-TDI) ${ }^{61}$ Hence, $298 \mathrm{~K}$ is the most suitable temperature for the removal of all the studied parabens using IL-MNP- $\beta C D-T D I$.

3.2.6 Effect of sorbent dosage. The results depicted in Fig. 5 indicate that the increase in the percentage removal is attributable to an increase in sorbent dosage up to $100 \mathrm{mg}$, but this was almost unchanged after $100 \mathrm{mg}$. This was mainly due to the increase in the number of active sites and surface areas during the adsorption process. ${ }^{62}$ Besides, the adsorption capacities displayed by IL-MNP- $\beta$ CD-TDI for all the studied parabens decreased as the dosage was increased. This signifies that all the active sites were entirely exposed and saturated at 


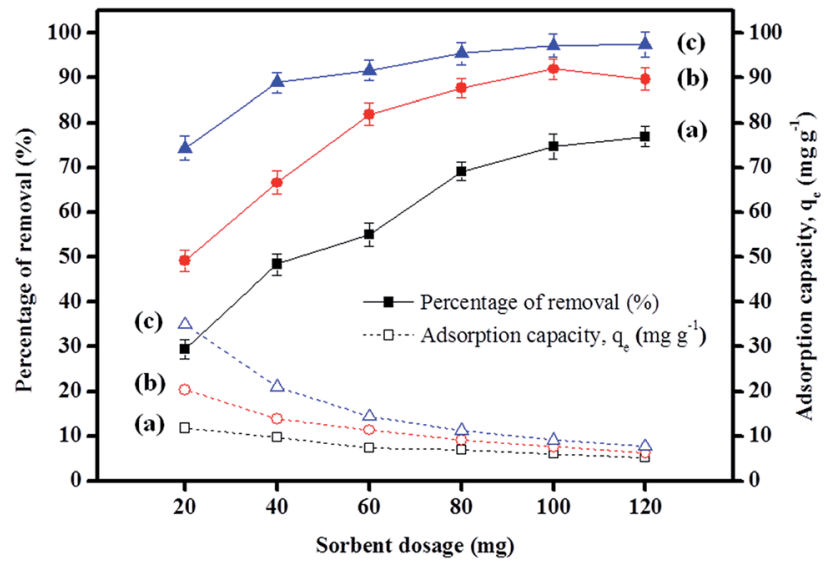

Fig. 5 Effect of sorbent dosage on the adsorption of (a) PP, (b) BP, and (c) $\operatorname{ArP}$ (conditions: initial concentration, $80 \mathrm{mg} \mathrm{L}^{-1}$; adsorption time, $80 \mathrm{~min}$; temperature, $298 \mathrm{~K}$ ).

a lower dosage, whereas only part of the active sites were utilized at a higher dosage owing to unsaturation of the adsorption sites via the adsorption reaction. ${ }^{63}$

\subsection{Adsorption study}

The adsorption process refers to the adhesion of elements to a surface that involves physisorption, chemisorption or electrostatic attraction. ${ }^{64}$ This method enhances water quality by removing toxic organic pollutants or chemical species, including organic compounds and trace metals. ${ }^{37,65}$
3.3.1 Adsorption kinetics. In order to determine the adsorption mechanism, the kinetic parameters were calculated via the adsorption of PP, BP, and ArP on IL-MNP- $\beta$ CD-TDI using the (a) pseudo-first-order, ${ }^{66}$ (b) pseudo-second-order, ${ }^{67,68}$ (c) Elovich, ${ }^{69}$ and (d) intraparticle diffusion ${ }^{70}$ models. Hence, to describe the validity and suitability of the adsorption kinetics, the normalized standard deviation $\Delta q(\%)$ and relative error (\%) were calculated using the following equations:

$$
\begin{gathered}
\Delta q(\%)=\sqrt{\frac{\left[\frac{q_{\exp }-q_{\mathrm{cal}}}{q_{\exp }}\right]^{2}}{N-1} \times 100} \\
\text { Relative error }(\%)=\frac{\left|q_{\exp }-q_{\mathrm{cal}}\right|}{q_{\exp }}
\end{gathered}
$$

where $N$ represents the number of data points, whereas $q_{\exp }$ and $q_{\mathrm{cal}}\left(\mathrm{mg} \mathrm{g}^{-1}\right)$ refer to the experimental and calculated adsorption capacities. According to the theory, a model fits better if the values of $\Delta q$ and the relative error (\%) are lower. ${ }^{69}$ Table 2 presents the fitting results for the adsorption of $\mathrm{PP}, \mathrm{BP}$, and ArP on IL-MNP-BCD-TDI, including the goodness and accuracy of the studied models, which were compared quantitatively in terms of the coefficients of determination $\left(R^{2}\right)$, normalized standard deviations, $\Delta q(\%)$, and relative errors (\%).

(a) Pseudo-first-order model. The Lagergren pseudo-firstorder equation has been widely applied in the adsorption of various solutes from aqueous solutions onto solid adsorbents. ${ }^{71}$ Lagergren developed a kinetic equation that describes the

\begin{tabular}{|c|c|c|c|c|}
\hline \multirow[b]{2}{*}{ Kinetic model } & \multirow[b]{2}{*}{ Parameter } & \multicolumn{3}{|c|}{ IL-MNP- $\beta C D-T D I$} \\
\hline & & PP & BP & ArP \\
\hline \multirow[t]{6}{*}{ Pseudo-first-order } & $q_{\mathrm{e}, \exp }\left(m g \mathrm{~g}^{-1}\right)$ & 3.0990 & 3.8721 & 4.6271 \\
\hline & $q_{\mathrm{e}, \mathrm{cal}}\left(\mathrm{mg} \mathrm{g}^{-1}\right)$ & 0.6295 & 0.2883 & 0.0812 \\
\hline & $k_{1}\left(\min ^{-1}\right)$ & 0.0343 & 0.0205 & 0.0177 \\
\hline & $\Delta q(\%)$ & 39.843 & 53.436 & 40.108 \\
\hline & Relative error (\%) & 79.687 & 92.554 & 98.245 \\
\hline & $R^{2}$ & 0.8510 & 0.8734 & 0.8609 \\
\hline \multirow[t]{6}{*}{ Pseudo-second-order } & $q_{\mathrm{e}, \mathrm{cal}}\left(\mathrm{mg} \mathrm{g}^{-1}\right)$ & 3.2331 & 3.8986 & 4.6104 \\
\hline & $k_{2}\left(\min ^{-1}\right)$ & 0.0852 & 0.2687 & 0.2860 \\
\hline & $H$ & 0.8906 & 4.0840 & 6.0792 \\
\hline & $\Delta q(\%)$ & 1.6355 & 0.2587 & 0.1364 \\
\hline & Relative error (\%) & 4.3272 & 0.6844 & 0.3609 \\
\hline & $R^{2}$ & 0.9984 & 0.9990 & 0.9997 \\
\hline \multirow[t]{6}{*}{ Elovich } & $q_{\mathrm{e}, \mathrm{cal}}\left(\mathrm{mg} \mathrm{g}^{-1}\right)$ & 3.0836 & 3.8214 & 4.6245 \\
\hline & $A$ & $2.4621 \times 10^{3}$ & $1.0289 \times 10^{5}$ & $3.5744 \times 10^{11}$ \\
\hline & $\beta$ & 4.4366 & 4.5579 & 7.1225 \\
\hline & $\Delta q(\%)$ & 0.2222 & 0.5856 & 0.0281 \\
\hline & Relative error (\%) & 0.4969 & 1.3094 & 0.0562 \\
\hline & $R^{2}$ & 0.9969 & 0.9197 & 0.9993 \\
\hline \multirow[t]{6}{*}{ Intraparticle diffusion } & $q_{\mathrm{e}, \mathrm{cal}}\left(\mathrm{mg} \mathrm{g}^{-1}\right)$ & 3.0451 & 3.8285 & 4.5537 \\
\hline & $K\left(\mathrm{mg} \mathrm{g}^{-1} \min ^{-1}\right)$ & 0.0832 & 0.0747 & 0.0376 \\
\hline & $C\left(\mathrm{mg} \mathrm{g}^{-1}\right)$ & 2.3009 & 3.1604 & 4.2174 \\
\hline & $\Delta q(\%)$ & 0.6574 & 0.4597 & 0.5996 \\
\hline & Relative error (\%) & 1.7393 & 1.1260 & 1.5863 \\
\hline & $R^{2}$ & 0.9564 & 0.7364 & 0.8201 \\
\hline
\end{tabular}

Table 2 Kinetic parameters for adsorption of PP, BP, and ArP on IL-MNP- $\beta C D-T D I$ 
adsorption of a liquid-solid system on the basis of the assumption that the rate of solute uptake over time is proportional to the variation in the saturation concentration and the amount of solid adsorbed over time. ${ }^{72}$ The equation is as follows:

$$
\frac{\mathrm{d} q_{t}}{\mathrm{~d}_{t}}=k_{1}\left(q_{\mathrm{e}}-q_{t}\right)
$$

where $q_{t}\left(\mathrm{mg} \mathrm{g}^{-1}\right)$ and $q_{\mathrm{e}}\left(\mathrm{mg} \mathrm{g}^{-1}\right)$ are the amounts of the analyte adsorbed on the synthesised adsorbent at time $t$ (min) and equilibrium, whereas $k_{1}\left(\mathrm{~min}^{-1}\right)$ refers to the rate constant of pseudo-first-order adsorption. When $q_{t}=0$ at $t=0$, eqn (5) can be integrated to give the following equations:

$$
\begin{gathered}
\log \left(q_{\mathrm{e}}-q_{t}\right)=\log q_{\mathrm{e}}-\frac{k_{1}}{2.303} t \\
\ln \left(q_{\mathrm{e}}-q_{t}\right)=\log q_{\mathrm{e}}-k_{1} t
\end{gathered}
$$

If the experimental data fit this kinetic model, a straight line can be obtained on a graph of $\ln \left(q_{\mathrm{e}}-q_{t}\right)$ versus $t$, and thus adsorption is followed by diffusion through a boundary. ${ }^{66}$ Besides, if the data fail to fit this equation, diffusion is excluded as the rate-determining step. The linear plot has $k_{1}$ as the slope, whereas $\log q_{\mathrm{e}}$ is the intercept.

(b) Pseudo-second-order model. The behaviour of a wide range of adsorption processes follows the pseudo-second-order model, which has chemisorption as the rate-determining step and adheres to the assumption that the adsorption mechanism relies on both the adsorbate and the adsorbent. ${ }^{66,68}$ According to Ho and McKay, if adsorption is in accordance with the pseudosecond-order model, the following equation gives the rate law: ${ }^{73}$

$$
\frac{\mathrm{d} q_{t}}{\mathrm{~d}_{t}}=k_{2}\left(q_{\mathrm{e}}-q_{t}\right)^{2}
$$

Upon integrating eqn (8) with $q_{t}=0$ at $t=0, k_{2}$ (g $\mathrm{mg}^{-1} \mathrm{~min}^{-1}$ ) is the equilibrium rate constant of the pseudosecond-order model, whereby the equation that is obtained can be rearranged to give:

$$
\frac{\mathrm{d} q_{t}}{\left(q_{\mathrm{e}}-q_{t}\right)}=k_{2} \mathrm{~d} t
$$

By integrating eqn (9) for values of $t=t$ and $q_{t}=q_{t}$, the integrated rate law is as follows:

$$
\frac{1}{\left(q_{\mathrm{e}}-q_{t}\right)}=\frac{1}{q_{\mathrm{e}}} k_{2} \mathrm{~d} t
$$

Moreover, eqn (10) can be rearranged into the following linear form:

$$
\frac{t}{q_{t}}=\frac{1}{k_{2} q_{\mathrm{e}}^{2}}+\frac{1}{q_{\mathrm{e}}} t
$$

from which the initial adsorption rate $\left(\mathrm{mg} \mathrm{g}^{-1} \min ^{-1}\right)$ is $h=$ $k_{2} q_{\mathrm{e}}{ }^{2}$. A plot of $\frac{t}{q_{t}}$ versus $t$ can be generated in which the values of $q_{\mathrm{e}}, k_{2}$, and $t_{1 / 2}$ are embedded; hence, a linear plot of $\frac{1}{q_{\mathrm{e}}}$ versus $\frac{1}{k_{2} q_{\mathrm{e}}^{2}}$ gives the slope and intercept of the pseudo-second-order model.

Table 2 shows that the pseudo-second-order model appears to fit the adsorption mechanism for all the paraben compounds more closely in comparison with the other studied models, as shown by its excellent coefficient of determination, $R^{2}>0.998$ (Fig. 3), and lower $\Delta q$ values of $1.6355 \%$ (PP), $0.2587 \%$ (BP), and $0.1364 \%$ (ArP), with relative errors of $4.3272 \%$ (PP), $0.6844 \%$ (BP), and $0.3609 \%$ (ArP), respectively. In fact, these results can be confirmed by the closeness of the $q_{\text {cal }}$ and $q_{\exp }$ values in the pseudo-second-order model. In particular, the adsorption process undertaken in this study for all the parabens was controlled by chemisorption, ${ }^{74}$ which involves valence sharing or the exchange of electrons $s^{37,75}$ and is based on the assumption that the adsorption mechanism depends on both the adsorbate and the adsorbent. ${ }^{6,68}$ Besides, the pseudo-second-order adsorption rate constants of the paraben compounds $\left(k_{2}\right)$ and the initial sorption rates $(h)$ on IL-MNP- $\beta C D$-TDI are in the order of $\operatorname{ArP}>$ BP $>$ PP.

(c) Elovich model. In an attempt to prove that the adsorption process was indeed based on chemisorption, the Elovich model was incorporated in this study. The Elovich equation is expressed as follows: ${ }^{69}$

$$
q_{t}=\frac{1}{\beta} \ln (\alpha \beta)+\frac{1}{\beta} \ln t
$$

where $\alpha$ is the initial sorption rate $\left(\mathrm{mg} \mathrm{g}^{-1} \mathrm{~min}^{-1}\right)$, whereas $\beta$ denotes the extended surface coverage and the activation energy for chemisorption $\left(\mathrm{g} \mathrm{mg}^{-1}\right)$. A linear plot of $q_{\mathrm{t}}$ versus $\ln t$ has $\frac{1}{\beta}$ and $\frac{1}{\beta} \ln (\alpha \beta)$ as the slope and intercept, respectively.

The exceptional coefficient of determination, $R^{2}>0.9$, seemed to fit a linear Elovich plot well. Moreover, in determining the occurrence of chemisorption, the $\Delta q$ values and relative errors appeared to be lower, which indicated a difference in values between $q_{\text {cal }}$ and $q_{\text {exp }}$. The values of $\beta$, on the other hand, signify the number of sites available for the adsorption process. Furthermore, $\alpha$ represents the adsorbed quantity when $\ln t=0$, i.e., when $t$ is $1 \mathrm{~min} .{ }^{76}$ Hence, the models fit the kinetic data for the adsorption of all the studied parabens in the following order: pseudo-second-order $>$ Elovich $>$ pseudofirst-order kinetics.

(d) Weber-Morris model. Weber-Morris intraparticle diffusion is defined by the equation below:

$$
q_{t}=K t^{0.5}+c
$$

where $K$ refers to the intraparticle diffusion rate constant (mg $\left.\mathrm{g}^{-1} \min ^{1 / 2}\right)$ and $c$ represents the intercept $\left(\mathrm{mg} \mathrm{g}^{-1}\right)$. The parameters $K$ and $c$ are determined from a linear plot of $q_{t}$ versus $t^{1 / 2}$. From the data presented in Fig. 6 , the line does not pass through the origin, which thus points out that intraparticle diffusion is not the rate-determining step but is indicative of some form of boundary layer within which another kinetic 


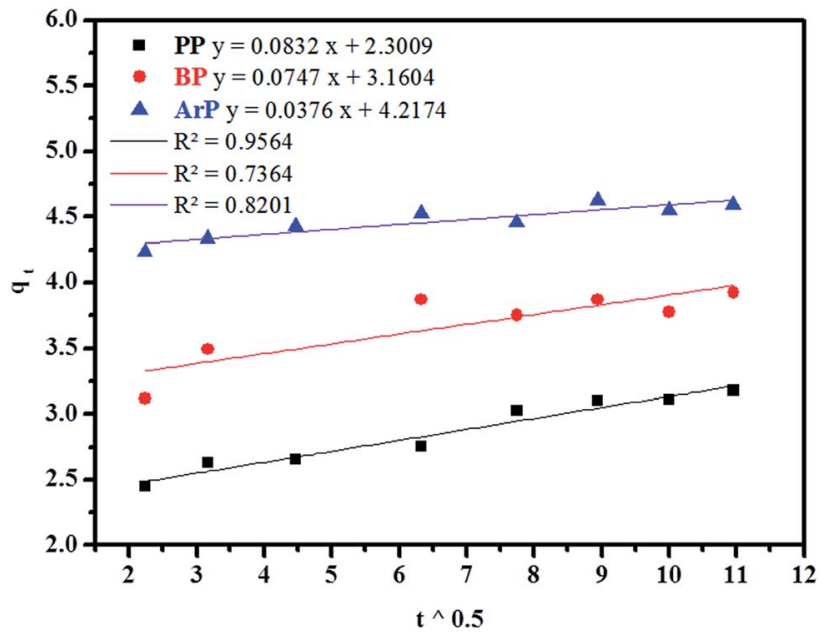

Fig. 6 Kinetic intraparticle diffusion model for the adsorption of PP, $B P$, and ArP on IL-MNP- $\beta C D-T D I$.

model that describes the adsorption rate may operate simultaneously. ${ }^{70}$ The value of $c$ suggests the boundary layer thickness that corresponds to a better adsorption mechanism for all the studied parabens. The greater is the intercept, for example, $2.3009 \mathrm{mg} \mathrm{g}^{-1}$ (PP), $3.1604 \mathrm{mg} \mathrm{g}^{-1}$ (BP), and $4.2174 \mathrm{mg} \mathrm{g}^{-1}$ (ArP), the greater is the boundary layer effect.

3.3.2 Adsorption isotherms. Adsorption isotherms describe the equilibrium adsorption of an adsorbate on an adsorbent's surface at a given constant temperature and $\mathrm{pH}$. Specifically, an isotherm is represented by plotting the equilibrium concentration of a compound on the adsorbent as a function of its equilibrium concentration in solution. ${ }^{77}$ In brief, an adsorption isotherm represents the interaction between the adsorbate and the adsorbent. Over the years, many equilibrium isotherm models have been designed to study the mechanism of these interactions. As such, this study incorporated three main isotherms, namely, the (a) Langmuir, (b) Freundlich, and (c) Temkin models. Non-linear equations have to be transformed into equations that are linear so as to obtain a linear fitting value of $R^{2}$. The $R^{2}$ value that is closest to unity indicates that an isotherm model provides the best fit to the experimental data. The experimental equilibrium data for the adsorption of PP, BP, and ArP on IL-MNP-BCD-TDI under the best conditions at three different temperatures $(298 \mathrm{~K}, 318 \mathrm{~K}$, and $338 \mathrm{~K}$ ) are tabulated in Table 3.

(a) Langmuir model. The Langmuir isotherm has been widely used to calculate the adsorption capacity for pollutants. Langmuir developed an equilibrium isotherm to reveal the correlation between the amount of gas adsorbed on the surface and the pressure of the gas. ${ }^{78}$ According to Langmuir, several assumptions that apply to this model are: (1) adsorption only takes place at specific homogeneous sites within the adsorbent, i.e., all surface adsorption sites have equal adsorption energies; (2) in adsorption, there are a finite adsorption capacity and a saturation point; and (3) adsorption of an adsorbate is directly proportional to its concentration in solution. The linear form of the Langmuir isotherm equation is represented as follows:

$$
\frac{1}{q_{\mathrm{e}}}=\frac{1}{b q_{\mathrm{m}}}+\frac{C_{\mathrm{e}}}{q_{\mathrm{m}}}
$$

where $C_{\mathrm{e}}\left(\mathrm{mg} \mathrm{L}^{-1}\right)$ is the equilibrium concentration of the adsorbate, $q_{\mathrm{e}}\left(\mathrm{mg} \mathrm{g}^{-1}\right)$ refers to the adsorption capacity at equilibrium, and $q_{\mathrm{m}}\left(\mathrm{mg} \mathrm{g}^{-1}\right)$ and $b\left(\mathrm{~L} \mathrm{mg}^{-1}\right)$ are the Langmuir constants related to the adsorption capacity and rate of adsorption, respectively. Besides, $q_{\mathrm{m}}$ and $b$ can be determined from a linear plot of $C_{\mathrm{e}} / q_{\mathrm{e}}$ versus $C_{\mathrm{e}}$, which has a slope of $\frac{1}{q_{\mathrm{m}}}$ and an intercept of $\frac{1}{b q_{\mathrm{m}}}$. Moreover, the dimensionless separation

Table 3 Details of isotherm constants for various adsorption isotherms for the adsorption of PP, BP, and ArP on IL-MNP- $\beta C D-T D I$

Isotherm model

\begin{tabular}{|c|c|c|c|c|}
\hline \multirow[b]{3}{*}{ Analyte } & \multicolumn{4}{|l|}{ Isotherm model } \\
\hline & \multirow[b]{2}{*}{ Parameter } & \multicolumn{3}{|c|}{ IL-MNP- $\beta C D-T D I$} \\
\hline & & $298 \mathrm{~K}$ & $318 \mathrm{~K}$ & $338 \mathrm{~K}$ \\
\hline \multicolumn{5}{|c|}{ Langmuir } \\
\hline \multirow[t]{4}{*}{ PP } & $q_{\mathrm{m}}\left(\mathrm{mg} \mathrm{g}^{-1}\right)$ & 18.48 & 18.12 & 15.63 \\
\hline & $b\left(\mathrm{~L} \mathrm{mg}^{-1}\right)$ & 0.0658 & 0.0216 & 0.0130 \\
\hline & $R_{\mathrm{L}}$ & 0.1627 & 0.3719 & 0.4959 \\
\hline & $R^{2}$ & 0.9512 & 0.9770 & 0.7522 \\
\hline \multirow[t]{4}{*}{$\mathrm{BP}$} & $q_{\mathrm{m}}\left(\mathrm{mg} \mathrm{g}^{-1}\right)$ & 28.49 & 25.13 & 19.08 \\
\hline & $b\left(\mathrm{~L} \mathrm{mg}^{-1}\right)$ & 0.0717 & 0.0414 & 0.0376 \\
\hline & $R_{\mathrm{L}}$ & 0.1505 & 0.2348 & 0.2526 \\
\hline & $R^{2}$ & 0.9589 & 0.9898 & 0.9870 \\
\hline \multirow[t]{4}{*}{$\operatorname{ArP}$} & $q_{\mathrm{m}}\left(\mathrm{mg} \mathrm{g}^{-1}\right)$ & 50.25 & 46.73 & 31.55 \\
\hline & $b\left(\mathrm{~L} \mathrm{mg}^{-1}\right)$ & 0.1004 & 0.0490 & 0.0463 \\
\hline & $R_{\mathrm{L}}$ & 0.1065 & 0.1963 & 0.2054 \\
\hline & $R^{2}$ & 0.9801 & 0.9709 & 0.9850 \\
\hline \multicolumn{5}{|c|}{ Freundlich } \\
\hline \multirow[t]{5}{*}{ PP } & $q_{\mathrm{m}}\left(\mathrm{mg} \mathrm{g}^{-1}\right)$ & 17.03 & 13.26 & 8.92 \\
\hline & $K_{\mathrm{F}}\left[\left(\mathrm{mg} \mathrm{g}^{-1}\right)\left(\mathrm{L} \mathrm{mg}^{-1}\right)^{1 / n_{\mathrm{F}}}\right]$ & 2.1145 & 0.5338 & 0.2689 \\
\hline & $n_{\mathrm{F}}$ & 2.1004 & 1.3641 & 1.2514 \\
\hline & $1 / n_{\mathrm{F}}$ & 0.4761 & 0.7331 & 0.7991 \\
\hline & $R^{2}$ & 0.9966 & 0.9809 & 0.9572 \\
\hline \multirow[t]{5}{*}{$\mathrm{BP}$} & $q_{\mathrm{m}}\left(\mathrm{mg} \mathrm{g}^{-1}\right)$ & 30.86 & 24.42 & 17.02 \\
\hline & $K_{\mathrm{F}}\left[\left(\mathrm{mg} \mathrm{g}^{-1}\right)\left(\mathrm{L} \mathrm{mg}^{-1}\right)^{1 / n_{\mathrm{F}}}\right]$ & 2.7714 & 1.3993 & 1.0464 \\
\hline & $n_{\mathrm{F}}$ & 1.8182 & 1.5326 & 1.5711 \\
\hline & $1 / n_{\mathrm{F}}$ & 0.5500 & 0.6525 & 0.6365 \\
\hline & $R^{2}$ & 0.9955 & 0.9781 & 0.9829 \\
\hline \multirow[t]{5}{*}{$\operatorname{ArP}$} & $q_{\mathrm{m}}\left(\mathrm{mg} \mathrm{g}^{-1}\right)$ & 87.67 & 59.05 & 34.06 \\
\hline & $K_{\mathrm{F}}\left[\left(\mathrm{mg} \mathrm{g}^{-1}\right)\left(\mathrm{L} \mathrm{mg}^{-1}\right)^{1 / n_{\mathrm{F}}}\right]$ & 4.8239 & 2.5734 & 1.8256 \\
\hline & $n_{\mathrm{F}}$ & 1.5110 & 1.3986 & 1.4975 \\
\hline & $1 / n_{\mathrm{F}}$ & 0.6618 & 0.7150 & 0.6678 \\
\hline & $R^{2}$ & 0.9865 & 0.9700 & 0.9606 \\
\hline \multicolumn{5}{|l|}{ Temkin } \\
\hline \multirow[t]{4}{*}{$\mathrm{PP}$} & $B$ & 3.0564 & 3.1254 & 2.3505 \\
\hline & $K_{\mathrm{T}}\left(\mathrm{L} \mathrm{mg}^{-1}\right)$ & 1.4711 & 0.3877 & 0.2954 \\
\hline & $b_{\mathrm{T}}\left(\mathrm{kJ} \mathrm{mol}^{-1}\right)$ & 810.62 & 845.92 & 1195.5 \\
\hline & $R^{2}$ & 0.9074 & 0.9605 & 0.9072 \\
\hline \multirow[t]{4}{*}{ BP } & $B$ & 4.9155 & 4.6864 & 3.6612 \\
\hline & $K_{\mathrm{T}}\left(\mathrm{L} \mathrm{mg}^{-1}\right)$ & 1.3806 & 0.6428 & 0.5440 \\
\hline & $b_{\mathrm{T}}\left(\mathrm{kJ} \mathrm{mol}^{-1}\right)$ & 504.03 & 564.15 & 767.54 \\
\hline & $R^{2}$ & 0.9283 & 0.9731 & 0.9642 \\
\hline \multirow[t]{4}{*}{$\operatorname{ArP}$} & $B$ & 8.7073 & 8.0738 & 5.9534 \\
\hline & $K_{\mathrm{T}}\left(\mathrm{L} \mathrm{mg}^{-1}\right)$ & 1.8765 & 0.8944 & 0.7054 \\
\hline & $b_{\mathrm{T}}\left(\mathrm{kJ} \mathrm{mol}^{-1}\right)$ & 284.54 & 327.46 & 472.02 \\
\hline & $R^{2}$ & 0.9455 & 0.9648 & 0.9833 \\
\hline
\end{tabular}


factor $\left(R_{\mathrm{L}}\right)$ can be calculated to determine if the adsorption process is favourable, as shown in the equation below, where $C_{\mathrm{o}}$ $\left(\mathrm{mg} \mathrm{L}^{-1}\right)$ represents the initial capacity for the adsorbate at equilibrium:

$$
R_{\mathrm{L}}=\frac{1}{1+b C_{\mathrm{o}}}
$$

$R_{\mathrm{L}}=0$ for irreversible adsorption, $0<R_{\mathrm{L}}<1$ for favourable adsorption, and $R_{\mathrm{L}}=1$ for linear adsorption, whereas $R_{\mathrm{L}}>1$ for unfavourable equilibrium.

Basically, the Langmuir model is applied to monolayer adsorption on a homogeneous system. As for this study, the adsorption process is assumed to follow the Langmuir model because the model appears to fit the experimental data rather well with exceptional coefficients of determination $\left(R^{2}>0.95\right)$ for all the studied parabens, except for PP at $338 \mathrm{~K}$, which gave a value of 0.7522 . This result reflects the lack of hydrophobicity in $\mathrm{PP}$, and hence its interaction with the $\beta \mathrm{CD}$ cavity was disregarded, primarily owing to the higher temperature (338 K), at which the analyte failed to remain in solution any longer and was subjected to degradation. The maximum monolayer adsorption $\left(q_{\mathrm{m}}\right)$ was $50.251 \mathrm{mg} \mathrm{g}^{-1}$ for the adsorption of ArP, with an $R_{\mathrm{L}}$ value of 0.1065 at $298 \mathrm{~K}$. The $R_{\mathrm{L}}$ values that were obtained for all the studied parabens seem favourable at all the studied temperatures as they exceeded zero but were less than unity $\left(0<R_{\mathrm{L}}<1\right)$.

(b) Freundlich model. The correlation between the adsorbate and the adsorbent at equilibrium was estimated from the coefficient of determination $\left(R^{2}\right)$, which indicated a better linear form that fitted the Freundlich isotherm model, followed by the Langmuir isotherm model. In fact, the Freundlich equation describes the adsorption equilibrium. This model is employed for adsorption on heterogeneous surfaces with various classes of adsorption sites, together with various energy levels of adsorption. The Freundlich equation is expressed as follows: ${ }^{73}$

$$
q_{\mathrm{m}}=K_{\mathrm{F}} C_{\mathrm{o}}^{\frac{1}{n_{\mathrm{F}}}}
$$

where $q_{\mathrm{m}}$ is the equilibrium concentration of the adsorbate on the adsorbent $\left(\mathrm{mg} \mathrm{g}^{-1}\right), C_{\mathrm{o}}$ refers to the initial equilibrium concentration of the adsorbate in solution $\left(\mathrm{mg} \mathrm{L}^{1}\right), K_{\mathrm{F}}$ denotes the Freundlich constant associated with multilayer adsorption capacity, and $\frac{1}{n_{\mathrm{F}}}$ represents the heterogeneity factor, where $n_{\mathrm{F}}$ is determined from the deviation from linear adsorption (adsorption is directly proportional to the concentration of the solution). ${ }^{66}$ From eqn (16), the following equation can be expressed in a linear form:

$$
\log q_{\mathrm{e}}=\log K_{\mathrm{F}}+\frac{1}{n_{\mathrm{F}}} \log C_{\mathrm{e}}
$$

Thus, the experimental data plotted as $\log q_{\mathrm{e}}$ versus $\ln C_{\mathrm{e}}$ were used to determine the intercept $K_{\mathrm{F}}$ and the slope $\frac{1}{n_{\mathrm{F}}}$. The value of $\frac{1}{n_{\mathrm{F}}}$ suggests the process of the adsorption system. ${ }^{79}$ Besides, Tseng and $\mathrm{Wu}$ asserted that if $n_{\mathrm{F}}<1$ the process is chemical adsorption (unfavourable), whereas $n_{\mathrm{F}}=1$ indicates linear adsorption, and $n_{\mathrm{F}}>1$ refers to favourable physical adsorption.

The adsorption of PP, BP, and ArP on IL-MNP- $\beta$ CD-TDI demonstrated that the $R^{2}$ value in the Freundlich model exceeded 0.95 at all the studied temperatures. This implies that the adsorption process on IL-MNP- $\beta$ CD-TDI involves a heterogeneous surface with many cavities (CD), isocyanate groups, and imidazolium rings. ${ }^{37}$ Moreover, $K_{\mathrm{F}}$ and $n_{\mathrm{F}}$ are the constants of the Freundlich isotherm that correspond to the adsorption capacity and intensity of the adsorbent, respectively. Hence, the significant decrease in the adsorption capacity $\left(K_{\mathrm{F}}\right)$ for all the studied parabens as the temperature rose indicates that the adsorption was exothermic, i.e., 2.1145-0.2689 (PP), 2.77141.0464 (BP), and 4.8239-1.8256 (ArP), as listed in Table 3. In addition, in order to prove that the adsorption process in this study is indeed favourable at lower temperatures, the intensity of the adsorbent $\left(n_{\mathrm{F}}\right)$ was calculated from the Freundlich model, whereby the results appeared to be in the range of $1<n_{\mathrm{F}}<10$ for the adsorption of all the studied parabens.

On the other hand, the sorption of ArP seemed to be better than that of BP, followed by that of PP, on IL-MNP- $\beta C D-T D I$, and this phenomenon may be explained by the inclusion effect and the $\pi-\pi$ interactions between the adsorbent and the adsorbates. Furthermore, the hydrophobicity of parabens depends on the alkyl chain in the parabens, whereby a longer alkyl chain results in higher hydrophobicity. Besides, the ability of $\beta C D$ to form a stable inclusion complex relies on the hydrophobicity of the analyte, which resulted in higher affinity for ArP owing to its higher hydrophobicity in comparison with that of BP, followed by that of PP. In addition, $\pi-\pi$ interactions occur via the imidazolium rings in the IL and the double bonds (aromatic rings) in the parabens. Because ArP possesses more double bonds, the interaction of ArP is stronger in comparison with that of BP, followed by that of PP. In brief, the order of the strength of the adsorption process on IL-MNP- $\beta$ CD-TDI is ArP $>$ BP $>$ PP, which corresponds to the exothermic nature of the process ( $298 \mathrm{~K}>318 \mathrm{~K}>338 \mathrm{~K}$ ).

(c) Temkin model. Temkin and Pyzhev incorporated the effect of some indirect and adsorbate/adsorbent interactions into the adsorption isotherm. ${ }^{80}$ It was also suggested that the heat of adsorption for all molecules in the adsorbent surface layer decreases linearly with the coverage. Adsorption is further characterized by a uniform distribution of binding energies up to the maximum binding energy. The Temkin isotherm can be expressed in a linear form, as given in the following equation:

$$
q_{\mathrm{e}}=\beta \ln K T+\beta \ln C_{\mathrm{e}}
$$

where $q_{\mathrm{e}}=\frac{R T}{b_{\mathrm{T}}}, \quad K_{\mathrm{T}}$ represents the equilibrium binding constant, which corresponds to the maximum binding energy $\left(\mathrm{L} \mathrm{mg}^{-1}\right)$, and $b_{\mathrm{T}}$ denotes the Temkin constant linked to the heat of adsorption. The constants $K_{\mathrm{T}}$ and $b_{\mathrm{T}}$ are determined from the intercept and slope, respectively, of a plot of $q_{\mathrm{e}}$ versus $\ln C_{\mathrm{e}}$.

The Temkin isotherm model also provides the best fit for the coefficient of determination $\left(R^{2}>0.90\right)$ for all the studied parabens at $298 \mathrm{~K}, 318 \mathrm{~K}$, and $338 \mathrm{~K}$. The Temkin constants $K_{\mathrm{T}}$ and $b_{\mathrm{T}}$ indicate the equilibrium binding constants that correspond 
Table 4 Thermodynamic parameters for all the studied parabens

\begin{tabular}{lllll}
\hline Analyte & $T(\mathrm{~K})$ & $\begin{array}{l}\Delta G^{\mathrm{o}} \\
\left(\mathrm{kJ} \mathrm{mol}^{-1}\right)\end{array}$ & $\begin{array}{l}\Delta H^{\mathrm{o}} \\
\left(\mathrm{J} \mathrm{mol}^{-1}\right)\end{array}$ & $\begin{array}{l}\Delta S^{\mathrm{o}} \\
\left(\mathrm{J} \mathrm{K}^{-1} \mathrm{~mol}^{-1}\right)\end{array}$ \\
\hline PP & 298 & 25.95 & -22.80 & -87.16 \\
& 318 & 27.70 & & \\
& 338 & 29.44 & & -61.28 \\
BP & 298 & 18.24 & -16.74 & \\
& 318 & 19.47 & & -89.60 \\
\multirow{4}{*}{ ArP } & 338 & 20.70 & & \\
& 298 & 26.67 & -28.22 & \\
& 318 & 28.46 & &
\end{tabular}

to the maximum binding energy $\left(\mathrm{L} \mathrm{mg}^{-1}\right)$ and the heat of sorption $\left(\mathrm{kJ} \mathrm{mol}^{-1}\right)$, respectively. The heat of sorption increased as the temperature rose, from 810.62 to $1195.50 \mathrm{~kJ} \mathrm{~mol}^{-1}$ (PP), from 504.03 to $767.54 \mathrm{~kJ} \mathrm{~mol}^{-1}$ (BP), and from 284.54 to $472.02 \mathrm{~kJ} \mathrm{~mol}^{-1}(\mathrm{ArP})$.

Therefore, in order to confirm the applicability of the isotherm models and to obtain some specific indications of the adsorption behaviour, it is crucial to identify the values of the coefficient of determination $\left(R^{2}\right)$ that correspond to all three linear forms of the studied models. Hence, an inspection of the $R^{2}$ values in Table 3 confirms that the equilibrium adsorption data display the best fit to the isotherm models in the order of Freundlich $>$ Langmuir $>$ Temkin for all the studied parabens (PP, BP, and ArP).

3.3.3 Adsorption thermodynamics. In order to determine the thermodynamic feasibility of the adsorption of PP, BP, and $\operatorname{ArP}$, the adsorption enthalpy $\left(\Delta H^{\circ}\right)$, change in entropy $\left(\Delta S^{\circ}\right)$, and Gibbs free energy $\left(\Delta G^{\circ}\right)$ were determined from the slope and intercept of a van't Hoff plot of $\ln K_{\mathrm{d}}$ versus $1 / T$ using eqn (20). In this case, $R$ denotes the universal gas constant $\left(\mathrm{kJ} \mathrm{mol}^{-1}\right.$ $\mathrm{K}^{-1}$ ) and $T$ is the temperature $(\mathrm{K})$, whereas $K_{\mathrm{d}}$ refers to the equilibrium constant $\left(\mathrm{m}^{3} \mathrm{~mol}^{-1}\right)$, which is determined from eqn (21), of which the results are tabulated in Table 4, whereas Fig. 7 presents the thermodynamic plots (van't Hoff plots) for the adsorption process.

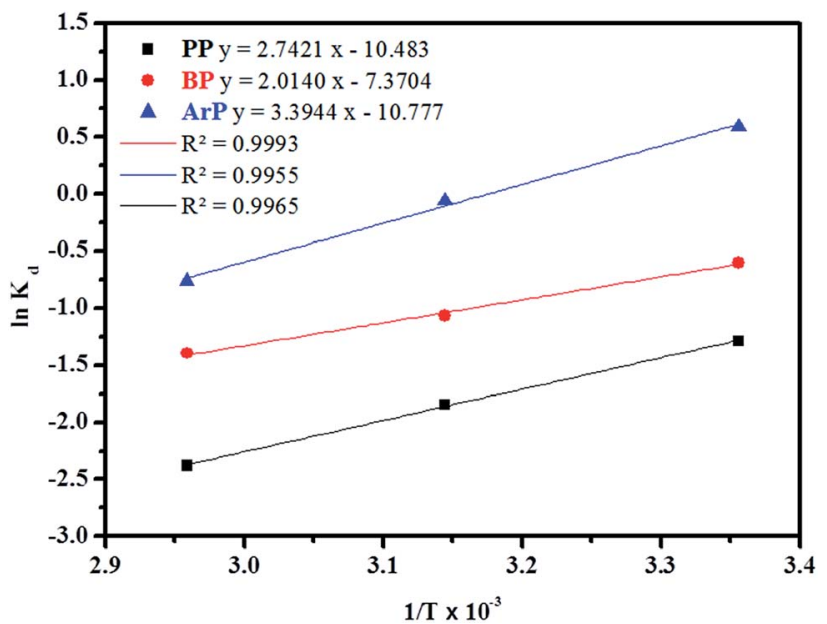

Fig. 7 Thermodynamic plots for the adsorption of PP, BP, and ArP on IL-MNP- $\beta C D-T D I$.

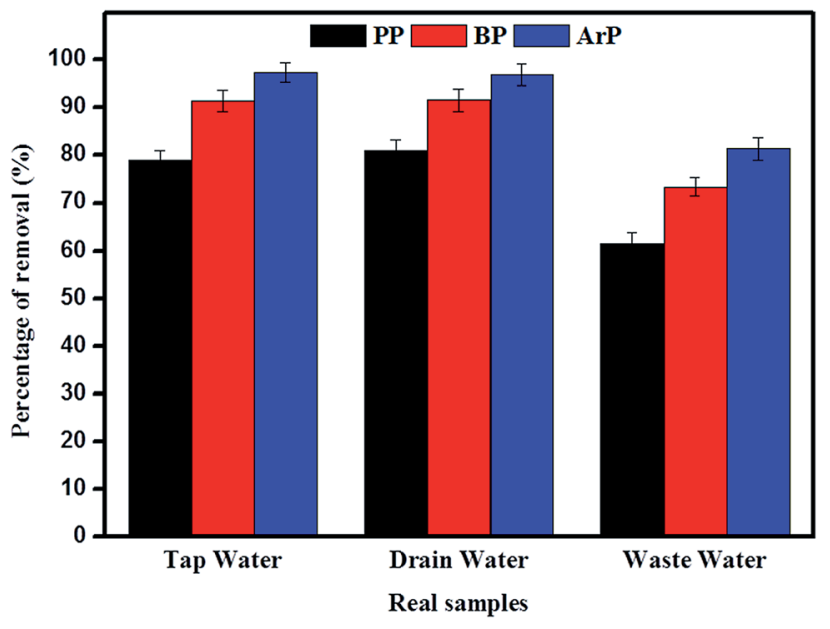

Fig. 8 Analysis of removal of PP, BP, and ArP from real samples (conditions: sorbent, $100 \mathrm{mg}$; initial concentration, $80 \mathrm{mg} \mathrm{L}^{-1}$. adsorption time, $80 \mathrm{~min}$; temperature, $298 \mathrm{~K}$ ).

$$
\begin{gathered}
\Delta G^{\mathrm{o}}=-R T \ln K_{\mathrm{d}} \\
\ln K_{\mathrm{d}}=\frac{\Delta S^{\mathrm{o}}}{R}-\frac{\Delta H^{\mathrm{o}}}{R T} \\
K_{\mathrm{d}}=\frac{q_{\mathrm{e}}(\text { equilibrium adsorbate on adsorbent })}{C_{\mathrm{e}}(\text { equilibrium adsorbate in solution })}
\end{gathered}
$$

From the van't Hoff plots, $\Delta H^{\mathrm{o}}$ was found to be negative $\left(-22.80,-16.74\right.$, and $-28.22 \mathrm{~J} \mathrm{~mol}^{-1}$ for PP, BP, and ArP, respectively), which confirms that the adsorption mechanism is indeed exothermic. Moreover, the negative values of $\Delta S^{\mathrm{o}}$ for all the studied parabens $\left(-87.17,-61.28\right.$, and $-89.60 \mathrm{~J} \mathrm{~K}^{-1} \mathrm{~mol}^{-1}$, respectively) are attributed to a decrease in the degree of freedom at the solid/solution interface in the adsorption process. ${ }^{81,82}$ Specifically, in order to confirm the spontaneity of the adsorption process, $\Delta G^{\mathrm{o}}$ should have a negative value at a given temperature.

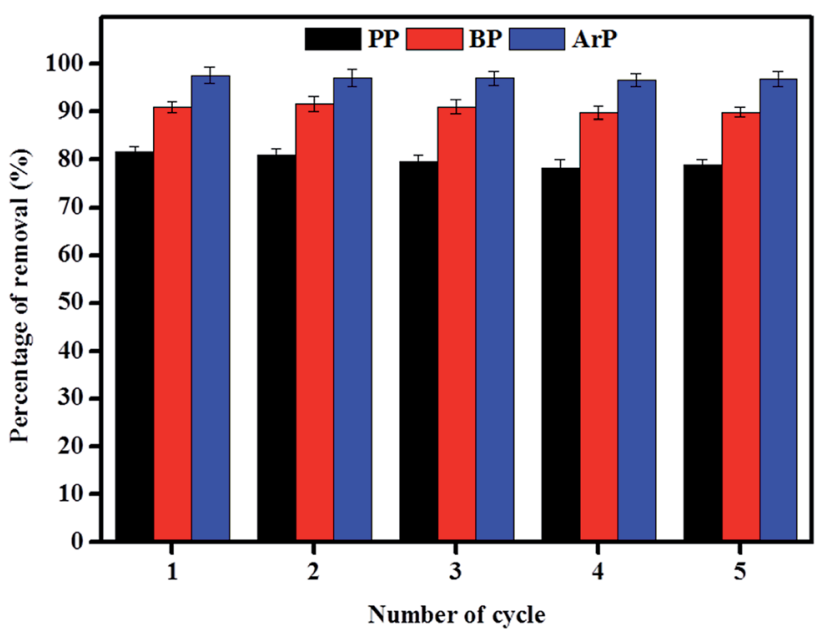

Fig. 9 Reusability of IL-MNP- $\beta C D-T D I$ for the adsorption of PP, BP, and $\operatorname{ArP}$ (conditions: sorbent, $100 \mathrm{mg}$; initial concentration, $80 \mathrm{mg} \mathrm{L}^{-1}$; adsorption time, $80 \mathrm{~min}$; temperature, $298 \mathrm{~K}$; desorption solvent, $5 \mathrm{~mL}$ $\mathrm{DI}$ and $5 \mathrm{~mL}$ ACN by vortexing). 
Table 5 Comparison of removal studies using various MSPE-based adsorbents

\begin{tabular}{|c|c|c|c|c|c|c|c|}
\hline Analytes & Adsorbent & $\begin{array}{l}\text { Sorbent } \\
\text { dosage }(\mathrm{mg})\end{array}$ & $\begin{array}{l}\text { Initial concentration } \\
\left(\mathrm{mg} \mathrm{L}^{-1}\right)\end{array}$ & $\begin{array}{l}\text { Reaction } \\
\text { time (min) }\end{array}$ & $\mathrm{pH}$ & $\begin{array}{l}\text { Maximum capacity, } \\
q_{\mathrm{e}}\left(\mathrm{mg} \mathrm{g}^{-1}\right)\end{array}$ & Reference \\
\hline DEHP & $\mathrm{Fe}_{3} \mathrm{O}_{4} @ \mathrm{P} 3 \mathrm{TArH}$ & 10 & 15 & 120 & 7 & 52.63 & 6 \\
\hline $\mathrm{Cu}^{2+}$ & $\mathrm{CM}-\beta-\mathrm{CD}-\mathrm{Fe}_{3} \mathrm{O}_{4}$ & 120 & $50-200$ & 30 & 6 & 47.20 & 30 \\
\hline DNA & {$\left[\mathrm{C}_{6} \mathrm{MIM}\right]-\mathrm{Fe}_{3} \mathrm{O}_{4}$} & 15 & 20 & 10 & 3 & 19.80 & 54 \\
\hline Fluoride & $\mathrm{Fe}_{3} \mathrm{O}_{4} @ \mathrm{Al}(\mathrm{OH})_{3} \mathrm{NPs}$ & 100 & 20 & 60 & 6.5 & 88.48 & 84 \\
\hline Reactive red 120 & $\mathrm{IL}-\mathrm{Fe}_{3} \mathrm{O}_{4}$ & 60 & 200 & 2 & 2.5 & 166.67 & 85 \\
\hline 4-(2-pyridylazo)resorcinol & & & & & & 49.26 & \\
\hline PP & IL-MNP- $\beta C D-T D I$ & 20 & 80 & 80 & 6 & 18.48 & This work \\
\hline $\mathrm{BP}$ & & & & & & 30.86 & \\
\hline ArP & & & & & & 87.67 & \\
\hline
\end{tabular}

Nevertheless, the thermodynamic parameters in this study appeared to be positive but low in terms of the value of $\Delta G^{\mathrm{o}}$ and thus indicated a feasible process that is non-spontaneous. ${ }^{83}$ Hence, the adsorption process for all the studied parabens can be concluded to be exothermic in nature and feasible, and could be spontaneous if the temperature is sufficiently low.

\subsection{Analysis of real samples}

The adsorption of PP, BP, and ArP was further investigated using real water samples. As illustrated in Fig. 8, the results for tap water and drain water appeared to be good enough in terms of removal of all the studied parabens. However, a decrease in the percentage removal was noted for industrial wastewater, which was mainly due to the matrix effect of the water itself. Moreover, industrial wastewater is known to contain many types of contaminants, which results in strong. competition between unknown analytes and the targeted analytes to saturate the surface of the materials. Therefore, the low removal efficiency for the industrial wastewater sample is no surprise, although the results are still above the acceptable levels of $60 \%, 70 \%$, and $80 \%$ for PP, BP, and ArP, respectively.

\subsection{Regeneration of adsorption activity displayed by IL-MNP- BCD-TDI}

The reuse of adsorbents has become an area of interest among many researchers, and this study area must be taken into consideration owing to its vast range of applications. Therefore, in order to investigate the possibility of the reuse of the material examined in this study, sequential adsorption-desorption experiments in batch mode were performed over five cycles. From the data presented in Fig. 9, an insignificant reduction in removal efficiency was observed after the material had been reused five times. This result indicates that IL-MNP-BCD-TDI is indeed an exceptional and promising material that can be used to remove paraben compounds. The cycling tests were, nonetheless, discontinued in this particular study owing to the limited time available as a result of the time taken for the material to dry, as well as its reusability, which had been tested on three types of paraben compounds (PP, BP, and ArP).

\subsection{Comparison of various magnetically based adsorbents}

As listed in Table 5, various magnetically based adsorbents were compared with various types of analytes, primarily because adsorption studies relating to paraben compounds are rare. The only adsorption study, which was reported by Forte et al. (2015), ${ }^{76}$ suggested that polyacrylonitrile (PAN) beads have been used to remove methyl paraben, but these are not a magnetically based adsorbent. Therefore, a comparison of the sorbent dosage (mg), initial concentration $\left(C_{\mathrm{o}}\right)$, equilibrium time, and maximum capacity $\left(q_{\mathrm{m}}\right)$ was undertaken, and these parameters were compared with those for various magnetically based adsorbents. Because IL-MNP- $\beta$ CD-TDI forms many types of interactions, a higher adsorption capacity was achieved by using a sorbent dosage of only $20 \mathrm{mg}$ with an equilibrium time of $80 \mathrm{~min}$.

\subsection{Adsorption behaviour of $\beta \mathrm{CD}-\mathrm{ArP}$ via formation of inclusion complex}

The formation of an inclusion complex can be confirmed by observing differences in chemical shifts $(\Delta \delta)$ based on specific nuclei in the host molecule, because such changes in the microenvironment have been believed to occur in CD in inclusion complexes. ${ }^{17}$ The boldface values in Table 6 signify the changes in chemical shifts that took place in the reaction. The noted downfield shift for the protons on the inner cavity of $\beta C D$, i.e., $\mathrm{H} 3$ and $\mathrm{H} 5$, corresponds to the encapsulation of the

Table 6 Chemical shifts $(\delta)$ of $\beta C D$, ArP, and $\beta C D$-ArP

\begin{tabular}{lllll}
\hline & $\beta C D(\delta)$ & $\operatorname{ArP}(\delta)$ & $\beta C D-A r P(\delta)$ & $\Delta \delta$ \\
\hline H1 & 4.832 & & 4.837 & +0.005 \\
H2 & 3.313 & & 3.313 & +0.000 \\
H3 & 3.634 & & 3.644 & $+\mathbf{0 . 0 1 0}$ \\
H4 & 3.351 & & 3.360 & $+\mathbf{0 . 0 0 9}$ \\
H5 & 3.620 & & 3.630 & $+\mathbf{0 . 0 1 0}$ \\
H6 & 3.648 & & 3.658 & $\mathbf{+ 0 . 0 1 0}$ \\
Ha-p & & 7.440 & 7.437 & $\mathbf{- 0 . 0 0 3}$ \\
Hb-p & & 7.393 & 7.399 & $\mathbf{+ 0 . 0 0 6}$ \\
Hc-p & & 7.347 & 7.345 & $\mathbf{0 . 0 0 2}$ \\
Hd-p & & 5.298 & 5.291 & -0.007 \\
He-p & & 7.863 & 7.856 & $\mathbf{- 0 . 0 0 7}$ \\
Hf-p & & 6.871 & 6.862 & $-\mathbf{0 . 0 0 9}$
\end{tabular}


aromatic ring of ArP, which penetrated into the cavity of CD. Moreover, the $\mathrm{H} 4$ and $\mathrm{H} 6$ protons of $\beta \mathrm{CD}$ are located on the outer part of the cavity but also exhibited a downfield shift, except that they did not exhibit substantial changes upon the encapsulation of the aromatic ring of ArP. Furthermore, changes in the microenvironment of ArP protons led to upfield shifts for Ha-p, Hc-p, Hd-p, He-p, and Hf-p, whereas a downfield shift was observed for Hb-p among these protons. These protons belong to the aromatic rings that are present in the structure of ArP, as illustrated in Fig. 10b. Hence, the ${ }^{1} \mathrm{H}$ signals of both $\beta C D$ and ArP strongly suggest the formation of inclusion complex interactions, which can be clearly observed in the ${ }^{1} \mathrm{H}$ NMR spectrum of $\beta C D$-ArP.

\subsection{Spectroscopic study}

3.8.1 Absorption spectrum of $\boldsymbol{\beta C D}$-ArP complex. A spectroscopic study was further conducted to confirm the formation of the inclusion complex, as well as to determine the binding capacity between $\mathrm{CD}$ and ArP. The absorption spectra of the

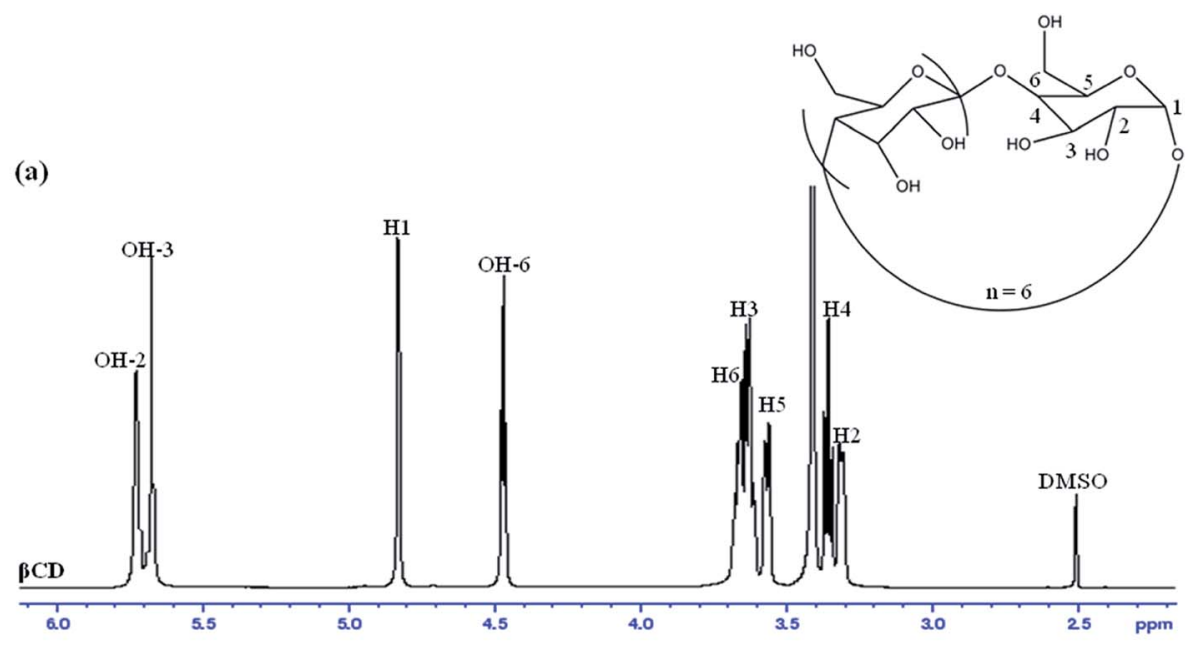

(b)

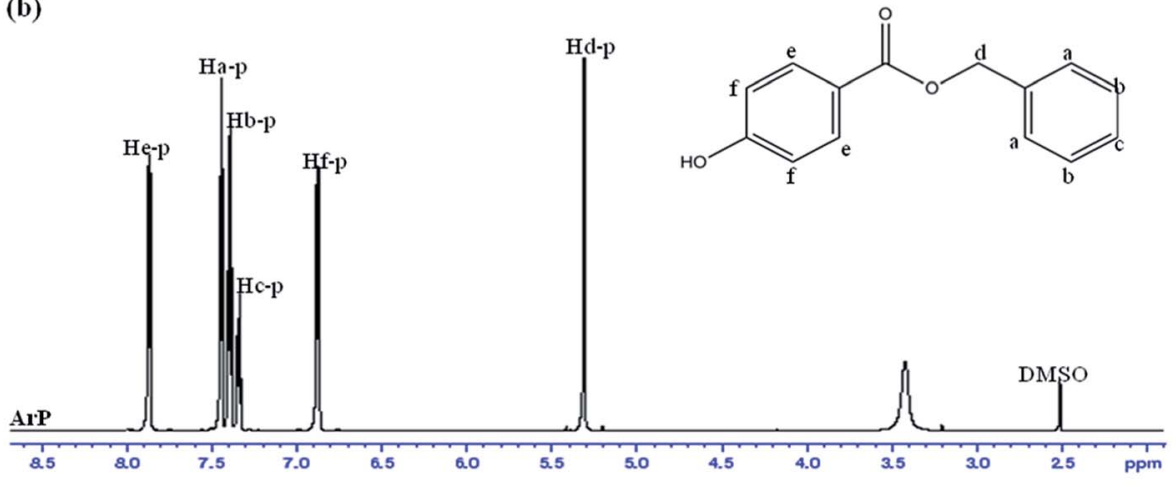

(c)

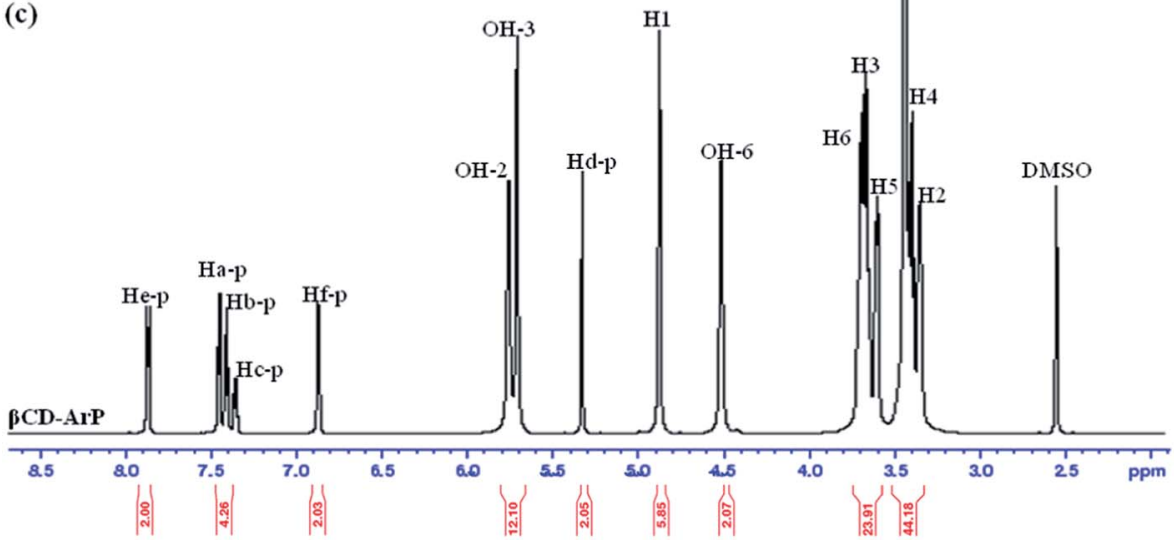

Fig. $10{ }^{1} \mathrm{H}$ NMR spectra of (a) $\beta C D$, (b) ArP, and (c) $\beta C D-A r P$. 


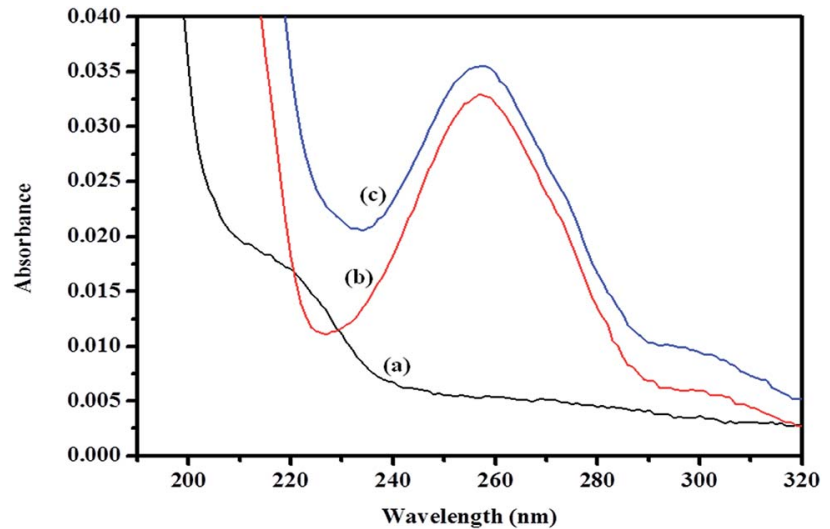

Fig. 11 Absorption spectra of (a) $\beta C D$, (b) ArP, and (c) the $\beta C D-A r P$ complex with [ArP]: $0.01 \mathrm{mM}$ and $[\beta C D]$ : $0.004 \mathrm{M}$ at a $\mathrm{pH}$ of 7 and $25^{\circ} \mathrm{C}$.

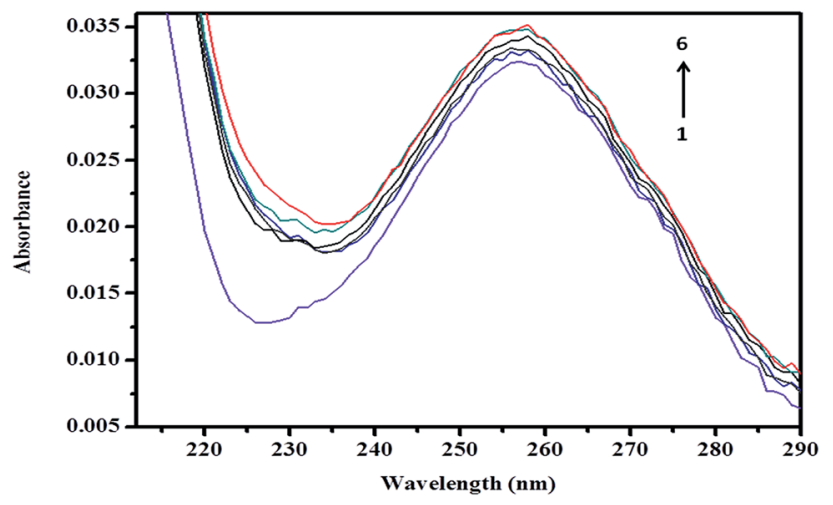

Fig. 12 Absorption spectra of $\operatorname{ArP}(0.01 \mathrm{mM})$ with various concentrations of $\beta C D$ at a pH of 7 and $25^{\circ} \mathrm{C}$. From line 1 to line $6: 0 \mathrm{M}, 0.004 \mathrm{M}$, $0.005 \mathrm{M}, 0.007 \mathrm{M}, 0.009 \mathrm{M}$, and $0.01 \mathrm{M}$.

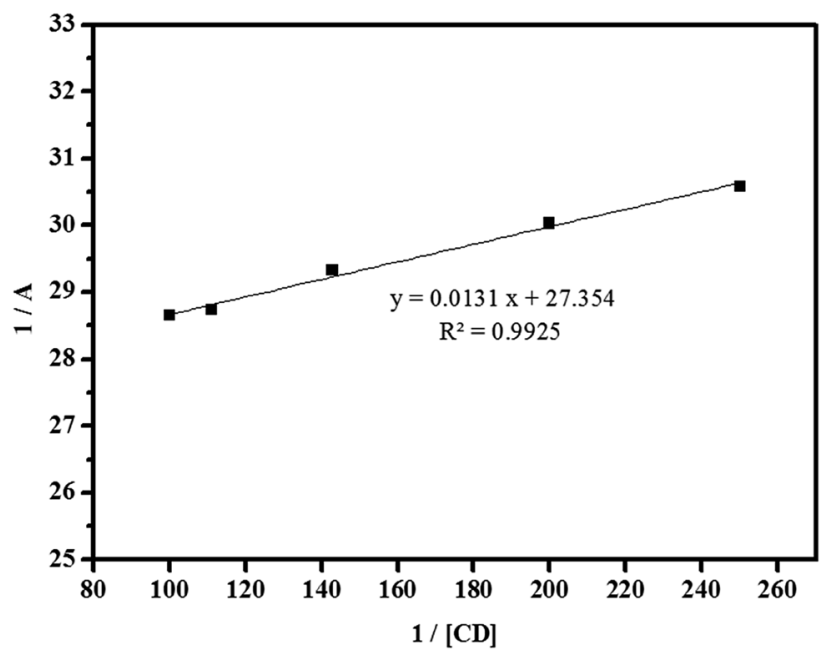

Fig. 13 Reciprocal plot of $1 / A$ versus $1 /[C D]$.

$\beta C D-A r P$ complex, ArP, and $\beta C D$ were recorded in accordance with the procedure described in Section 2.4.10. The results presented in Fig. 11 indicate that $\beta C D$ exhibited no absorption in the range from 240 to $320 \mathrm{~nm}$. In addition, the absorption spectrum of ArP seems rather similar to the spectrum of the $\beta C D-A r P$ complex, except that the absorbance of the inclusion complex appeared to be higher than that of ArP at every wavelength. Furthermore, the influence of the $\beta C D$ concentration on ArP was also studied, and the results are illustrated in Fig. 12. An increase in the $\beta C D$ concentration led to a distinct variation in absorption intensity. Upon penetrating into the $\beta C D$ cavity, the absorbance of the guest molecule was enhanced owing to the shielding of the excited species from non-radiative processes that occur in the bulk solution, together with an increase in the molar absorption coefficient of the inclusion complex. ${ }^{17}$

3.8.2 Stoichiometry of the complex and the formation constant. The formation constant, which is denoted as $K$, was calculated by dividing the slope by the intercept of the straight line obtained in a reciprocal plot, whereas the stoichiometric ratio of $\beta \mathrm{CD}$ to ArP was determined using the Benesi-Hildebrand equation: ${ }^{86}$

$$
\frac{1}{A}=\frac{1}{\varepsilon[\mathrm{G}]_{\mathrm{o}} K[\mathrm{CD}]}+\frac{1}{\varepsilon[\mathrm{G}]_{\mathrm{o}}}
$$

where $A$ is the absorbance of the targeted analyte at each $\beta C D$ concentration, $[\mathrm{G}]_{\mathrm{o}}$ refers to the initial concentration of the targeted analyte, $K$ denotes the apparent formation constant, $[\mathrm{CD}]$ represents the concentration of $\beta \mathrm{CD}$, and $\varepsilon$ is the molar absorptivity. Furthermore, the formation constant of the inclusion complex was determined by analysing the fluctuations observed in the absorption intensity with variations in the $\beta C D$ concentration. The formation constant, which is denoted as $K$, was calculated by dividing the slope by the intercept of the straight line obtained in a reciprocal plot (Fig. 13). The graph, which was plotted with $1 / A$ against $1 /[\mathrm{CD}]$, displays a good linear coefficient of determination, $R^{2}=0.9925$, and thus signifies that the stoichiometric ratio of the complex that was formed is $1: 1$. In fact, the apparent formation constant is $2.09 \times$ $10^{3} \mathrm{~mol} \mathrm{~L}^{-1}$.

\section{Conclusion}

In conclusion, IL-MNP- $\beta$ CD-TDI was successfully tested as an adsorbent for the adsorption of selected paraben compounds, namely, PP, BP, and ArP. Furthermore, both kinetic and isotherm analyses demonstrated that the pseudo-second-order and Freundlich models provided better correlations for the adsorption of $\mathrm{PP}, \mathrm{BP}$, and $\mathrm{ArP}$, with $R^{2}$ values ranging between 0.980 and 0.999 for both models. In addition, both analyses were found to indicate that the equilibrium time was $80 \mathrm{~min}$ with an initial concentration of $80 \mathrm{mg} \mathrm{L}^{-1}$ at room temperature (298 K). Moreover, the negative value of $\Delta H$ demonstrated the exothermic nature of the adsorption process for all the studied parabens. In addition, the adsorbent was further analysed using real water samples. IL-MNP- $\beta C D$-TDI was also tested for the reusability of the sorbent, and it was found that the sorbent could be reused up to five times. Finally, an adsorption mechanism is proposed by considering the inclusion complex and $\pi-$ $\pi$ interaction between $\beta \mathrm{CD}$ and ArP, as proven via experimental analysis of ${ }^{1} \mathrm{H}$ NMR spectra. Spectroscopic analysis also confirmed the formation of the inclusion complex by an 
enhancement in the absorbance of the guest molecule in UV-vis analysis. In brief, IL-MNP- $\beta$ CD-TDI appears to be a promising material for the treatment of parabens in water.

\section{Conflicts of interest}

All authors declare that there is no conflict of interest.

\section{Acknowledgements}

Grateful thanks to the scholarship provided by MyBrain 15 (MyMaster) from the Ministry of Higher Education (MOHE), Malaysia to one of the authors, Masrudin bin Md Yusoff. The authors would also like to thank the Universiti Sains Malaysia Research Grant (RUi: 1001/CIPPT/811322) and the Fundamental Research Grant Scheme (FRGS: 203/CIPPT/6711557) for their financial support to finish this study.

\section{References}

1 A. H. Lu, E. e. L. Salabas and F. Schüth, Angew. Chem., Int. Ed., 2007, 46, 1222-1244.

2 G. Giakisikli and A. N. Anthemidis, Anal. Chim. Acta, 2013, 789, 1-16.

3 N. Sanvicens and M. P. Marco, Trends Biotechnol., 2008, 26, 425-433.

4 S. K. M. Rozi, S. Bakhshaei, N. S. A. Manan and S. Mohamad, RSC Adv., 2016, 6, 87719-87729.

5 W. Wang, R. Ma, Q. Wu, C. Wang and Z. Wang, J. Chromatogr. A, 2013, 1293, 20-27.

6 S. N. A. Baharin, N. Muhamad Sarih and S. Mohamad, Polymers, 2016, 8, 117.

7 E. Tahmasebi, Y. Yamini, A. Mehdinia and F. Rouhi, J. Sep. Sci., 2012, 35, 2256-2265.

8 J. Ding, Q. Gao, D. Luo, Z.-G. Shi and Y.-Q. Feng, J. Chromatogr. A, 2010, 1217, 7351-7358.

9 H. Heidari and H. Razmi, Talanta, 2012, 99, 13-21.

10 Z. Du, M. Liu and G. Li, J. Sep. Sci., 2013, 36, 3387-3394.

11 M. Alcudia-León, R. Lucena, S. Cárdenas and M. Valcárcel, Microchem. J., 2013, 110, 643-648.

12 A. Mehdinia, F. Roohi and A. Jabbari, J. Chromatogr. A, 2011, 1218, 4269-4274.

13 T. D. Schladt, K. Schneider, H. Schild and W. Tremel, Dalton Trans., 2011, 40, 6315-6343.

14 Y. He and X. Shen, J. Photochem. Photobiol., A, 2008, 197, 253-259.

15 J. Szejtli, Chem. Rev., 1998, 98, 1743-1754.

16 K. P. Sambasevam, S. Mohamad, N. M. Sarih and N. A. Ismail, Int. J. Mol. Sci., 2013, 14, 3671-3682.

17 S. Mohamad, H. Surikumaran, M. Raoov, T. Marimuthu, K. Chandrasekaram and P. Subramaniam, Int. J. Mol. Sci., 2011, 12, 6329-6345.

18 J. Zhang, X. Shen and Q. Chen, Curr. Org. Chem., 2011, 15, 74-85.

19 W. Lu, X. Le, J. Zhang, Y. Huang and T. Chen, Chem. Soc. Rev., 2017, 46, 1284-1294.
20 E. Y. Ozmen, A. Sirit and M. Yilmaz, J. Macromol. Sci., Part A: Pure Appl. Chem., 2007, 44, 167-173.

21 Y. P. Chin, S. Mohamad and M. R. B. Abas, Int. J. Mol. Sci., 2010, 11, 3459-3471.

22 K. Salipira, R. Krause, B. Mamba, T. Malefetse, L. Cele and S. Durbach, Mater. Chem. Phys., 2008, 111, 218-224.

23 A. Romo, F. J. Penas, J. R. Isasi, I. X. Garcia-Zubiri and G. González-Gaitano, React. Funct. Polym., 2008, 68, 406-413.

24 S. M. Ng and R. Narayanaswamy, Sens. Actuators, B, 2009, 139, 156-165.

25 I. X. García-Zubiri, G. González-Gaitano and J. R. Isasi, J. Colloid Interface Sci., 2009, 337, 11-18.

26 M. Appell and M. A. Jackson, J. Inclusion Phenom. Macrocyclic Chem., 2010, 68, 117-122.

27 H. Cao, J. He, L. Deng and X. Gao, Appl. Surf. Sci., 2009, 255, 7974-7980.

28 A. Z. M. Badruddoza, G. S. S. Hazel, K. Hidajat and M. Uddin, Colloids Surf., A, 2010, 367, 85-95.

29 R. Li, S. Liu, J. Zhao, H. Otsuka and A. Takahara, Polym. Bull., 2011, 66, 1125-1136.

30 A. Z. M. Badruddoza, A. Tay, P. Tan, K. Hidajat and M. Uddin, J. Hazard. Mater., 2011, 185, 1177-1186.

31 H. Wang, Y. Zhou, Y. Guo, W. Liu, C. Dong, Y. Wu, S. Li and S. Shuang, Sens. Actuators, B, 2012, 163, 171-178.

32 A. R. Kiasat and S. Nazari, J. Mol. Catal. A: Chem., 2012, 365, 80-86.

33 A. Z. M. Badruddoza, Z. B. Z. Shawon, D. W. J. Tay, K. Hidajat and M. S. Uddin, J. Chem. Eng., 2013, 27, 69-73.

34 A. Z. M. Badruddoza, Z. B. Z. Shawon, W. J. D. Tay, K. Hidajat and M. S. Uddin, Carbohydr. Polym., 2013, 91, 322-332.

35 A. Gong, W. Ping, J. Wang and X. Zhu, Spectrochim. Acta, Part $A, 2014,122,331-336$.

36 L. Fan, M. Li, Z. Lv, M. Sun, C. Luo, F. Lu and H. Qiu, Colloids Surf., B, 2012, 95, 42-49.

37 M. Raoov, S. Mohamad and M. R. Abas, J. Hazard. Mater., 2013, 263, 501-516.

38 M. Raoov, S. Mohamad and M. R. Abas, Int. J. Mol. Sci., 2013, 15, 100-119.

39 M. Raoov, S. Mohamad, M. R. bin Abas and H. Surikumaran, Talanta, 2014, 130, 155-163.

40 W. Ping, H. Xu and X. Zhu, Biochem. Anal. Biochem., 2013, 2, 1000138.

41 W. Ping, X. Zhu and B. Wang, Anal. Lett., 2014, 47, 504-516. 42 X. Qin and X. Zhu, Anal. Lett., 2016, 49, 189-199.

43 P. Subramaniam, S. Mohamad and Y. Alias, Int. J. Mol. Sci., 2010, 11, 3675-3685.

44 A. B. McEwen, H. L. Ngo, K. LeCompte and J. L. Goldman, J. Electrochem. Soc., 1999, 146, 1687-1695.

45 J. L. Anderson, J. Ding, T. Welton and D. W. Armstrong, J. Am. Chem. Soc., 2002, 124, 14247-14254.

46 J. A. Ocaña-González, M. Villar-Navarro, M. Ramos-Payán, R. Fernández-Torres and M. A. Bello-López, Anal. Chim. Acta, 2015, 858, 1-15.

47 P. D. Darbre and P. W. Harvey, J. Appl. Toxicol., 2014, 34, 925938.

48 C. Haman, X. Dauchy, C. Rosin and J.-F. Munoz, Water Res., 2015, 68, 1-11. 
49 D. Błędzka, J. Gromadzińska and W. Wąsowicz, Environ. Int., 2014, 67, 27-42.

50 S. Sasi, M. P. Rayaroth, D. Devadasan, U. K. Aravind and C. T. Aravindakumar, J. Hazard. Mater., 2015, 300, 202-209.

51 M. M. Yusoff, M. Raoov, N. Yahaya and N. M. Salleh, RSC Adv., 2017, 7, 35832-35844.

52 S. Sinniah, S. Mohamad and N. S. Manan, Appl. Surf. Sci., 2015, 357, 543-550.

53 M. Karimi, A. M. Shabani and S. Dadfarnia, J. Braz. Chem. Soc., 2016, 27, 144-152.

54 M. Ghaemi and G. Absalan, Microchim. Acta, 2014, 181, 4553.

55 T. Chatzimitakos, C. Binellas, K. Maidatsi and C. Stalikas, Anal. Chim. Acta, 2016, 910, 53-59.

56 M. Bhaskar, P. Aruna, R. J. G. Jeevan and G. Radhakrishnan, Anal. Chim. Acta, 2004, 509, 39-45.

57 Z. Ma, Y. Guan and H. Liu, J. Polym. Sci., Part A: Polym. Chem., 2005, 43, 3433-3439.

58 K. S. Sing, Pure Appl. Chem., 1985, 57, 603-619.

59 A. Ismail, D. Adil, B. Erhan and S. Bekir, Chemosphere, 2005, 61, 1263-1272.

60 S. Bakhshaei, M. A. Kamboh, H. R. Nodeh, S. M. Zain, S. K. M. Rozi, S. Mohamad and I. A. M. Mohialdeen, $R S C$ Adv., 2016, 6, 77047-77058.

61 D. Bulgariu and L. Bulgariu, Bioresour. Technol., 2012, 103, 489-493.

62 E. N. El Qada, S. J. Allen and G. M. Walker, Ind. Eng. Chem. Res., 2006, 45, 6044-6049.

63 F. Y. Wang, H. Wang and J. W. Ma, J. Hazard. Mater., 2010, 177, 300-306.

64 Y. Wu, E. Hu, W. Dai, Z. Li, Y. Zhong and Y. Hu, RSC Adv., 2017, 7, 5093-5100.

65 G. Crini, Prog. Polym. Sci., 2005, 30, 38-70.

66 G. Crini, H. N. Peindy, F. Gimbert and C. Robert, Sep. Purif. Technol., 2007, 53, 97-110.

67 G. Crini, Dyes Pigm., 2008, 77, 415-426.
68 F. A. Pavan, S. L. Dias, E. C. Lima and E. V. Benvenutti, Dyes Pigm., 2008, 76, 64-69.

69 M. d. M. C. López, M. C. Pérez, M. S. D. García, J. M. L. Vilarino, M. V. G. Rodríguez and L. F. B. Losada, Anal. Chim. Acta, 2012, 721, 68-78.

70 M. Kilic, E. Apaydin-Varol and A. E. Pütün, J. Hazard. Mater., 2011, 189, 397-403.

71 H. Yuh-Shan, Scientometrics, 2004, 59, 171-177.

72 S. Lagergren, K. Sven. Vetenskapsakad. Handl., 1898, 24, 1-39.

73 Y.-S. Ho and G. McKay, Process Biochem., 1999, 34, 451-465.

74 I. Tan, A. Ahmad and B. Hameed, J. Hazard. Mater., 2009, 164, 473-482.

75 V. Vimonses, S. Lei, B. Jin, C. W. Chow and C. Saint, Chem. Eng. J., 2009, 148, 354-364.

76 M. Forte, L. Mita, R. Perrone, S. Rossi, M. Argirò, D. G. Mita, M. Guida, M. Portaccio, T. Godievargova and Y. Ivanov, Environ. Sci. Pollut. Res., 2016, 1-13.

77 A. Delle Site, J. Phys. Chem. Ref. Data, 2001, 30, 187-439.

78 I. Langmuir, J. Am. Chem. Soc., 1918, 40, 1361-1403.

79 R.-L. Tseng and F.-C. Wu, J. Hazard. Mater., 2008, 155, 277287.

80 M. Temkin and V. Pyzhev, Acta Physicochim. URSS, 1940, 12, 217-222.

81 Y. A. Aydın and N. D. Aksoy, Chem. Eng. J., 2009, 151, 188194.

82 T. A. Saleh, A. Sarı and M. Tuzen, J. Mol. Liq., 2016, 219, 937945.

83 S. Gueu, B. Yao, K. Adouby and G. Ado, Int. J. Environ. Sci. Technol., 2007, 4, 11-17.

84 X. Zhao, J. Wang, F. Wu, T. Wang, Y. Cai, Y. Shi and G. Jiang, J. Hazard. Mater., 2010, 173, 102-109.

85 G. Absalan, M. Asadi, S. Kamran, L. Sheikhian and D. M. Goltz, J. Hazard. Mater., 2011, 192, 476-484.

86 H. Y. Wang, J. Han and X. G. Feng, Spectrochim. Acta, Part A, 2007, 66, 578-585. 